\title{
Recursion operators, conservation laws and integrability conditions for difference equations
}

\author{
Alexander V. Mikhailov^, Jing Ping Wang ${ }^{\dagger}$ and Pavlos Xenitidis * \\ * Applied Mathematics Department, University of Leeds, UK \\ $\dagger$ School of Mathematics and Statistics, University of Kent, UK
}

\begin{abstract}
In this paper we make an attempt to give a consistent background and definitions suitable for the theory of integrable difference equations. We adapt a concept of recursion operator to difference equations and show that it generates an infinite sequence of symmetries and canonical conservation laws for a difference equation. Similar to the case of partial differential equations these canonical densities can serve as integrability conditions for difference equations. We have found the recursion operators for the Viallet and all ABS equations.
\end{abstract}

\section{Introduction}

The nature of integrability of partial differential equations is rather well understood [1, 2]. It can be related to the existence of Lax representations, Miura and Bäcklund transformations, bi-linear Hirota representations, multi-soliton solutions, the Painlevé property, bi-Hamiltonian structures, recursion operators and infinite hierarchies of symmetries and conservation laws. Although there is not any commonly accepted definition for integrability, the patterns of equations possessing some of the above listed properties coincide. In the symmetry approach the existence of an infinite hierarchy of symmetries and conservation laws is taken as a definition of integrability and the most complete results on classification of integrable equations are based on this property [3, 4, 5, 6, 7, 8, 9, The symmetry approach proved to be effective in the problem of classification of integrable differentialdifference equations [10, 11].

The theory of integrable difference (or discrete) equations is a relatively recent, but very active area of research. It inherited some methods and tools from the theory of integrable partial differential equations, see for instance [12, 13]. The property of multidimensional consistency, or consistency around the cube [14, has been proposed as the integrability criterion for quadrilateral difference equations 15, 16. All integrable affine-linear quadrilateral equations (satisfying a few symmetry conditions with respect to reflections of the three dimensional lattice) were classified by Adler, Bobenko and Suris [17, see also [18]. They produced a list of seven equations (up to point fractional-linear transformations), which will be referred as the ABS equations. Unfortunately, the method based on the property of multi-dimensional consistency cannot be directly applied to a given equation on a lattice. Indeed, to check the consistency around the cube one needs to know the equations corresponding to the sides of the cube.

Another characteristic property of an integrable equation is the vanishing of its algebraic entropy 
[19]. It has been used by Viallet to identify the equation

$$
\begin{aligned}
Q:= & a_{1} u_{0,0} u_{1,0} u_{0,1} u_{1,1}+a_{2}\left(u_{0,0} u_{1,0} u_{0,1}+u_{1,0} u_{0,1} u_{1,1}+u_{0,1} u_{1,1} u_{0,0}+u_{1,1} u_{0,0} u_{1,0}\right) \\
& +a_{3}\left(u_{0,0} u_{1,0}+u_{0,1} u_{1,1}\right)+a_{4}\left(u_{1,0} u_{0,1}+u_{0,0} u_{1,1}\right)+a_{5}\left(u_{0,0} u_{0,1}+u_{1,0} u_{1,1}\right) \\
& +a_{6}\left(u_{0,0}+u_{1,0}+u_{0,1}+u_{1,1}\right)+a_{7}=0
\end{aligned}
$$

where $a_{i}$ are free complex parameters [20]. By a point fractional-linear transformation equation (11) with a generic choice of parameters can be reduced to Adler's equation, also referred as the Q4 equation in the ABS classification. Therefore it is not surprising that the Viallet equation (1) has a hierarchy of symmetries [21]. All of the ABS equations can be obtained from the Viallet equation by a simple specialisation of parameters, it is a convenient way to represent the ABS equations. The obvious advantage of the Viallet approach is its applicability to a given equation.

The aim of this paper is to develop a theory of difference equations, based on the study of symmetries, conservation laws and (formal) recursion operators. By integrability of a difference equation we understand the existence of an infinite hierarchy of its symmetries. In this paper we consider quadrilateral equations $Q=0$, where $Q$ is assumed to be an irreducible affine-linear polynomial over $\mathbb{C}$. Symmetries, conservation laws, and actually all rational functions which make sense on solutions of the difference equation are elements of a difference field of fractions $\mathcal{F}_{Q}$ defined in Section 2. The affine-linearity of the equation enables us to introduce the elimination map $\mathcal{E}$, which reduces any element of $\mathcal{F}_{Q}$ to a rational function of dynamical variables. This map is quite a useful tool for computations and proofs, since two functions $f$ and $g$ of the lattice variables are equivalent modulo the difference equation if and only $\mathcal{E}(f)=\mathcal{E}(g)$. In Section 2 we give a self-contained set of basic definitions required in this paper.

In Section 3 we give a definition of a recursion operator for difference equations. The recursion operator acting on a seed symmetry of the difference equation generates an infinite hierarchy of symmetries of that difference equation. We show that the residues of the powers of the recursion operator are (canonical) densities of conservation laws for the corresponding difference equation. We can generate an infinite sequence of canonical conservation laws (both the densities and fluxes). Similar to the differential case (see [3, 4, 5]), these canonical conservation laws can be used as integrability conditions for a difference equation. They are the necessary conditions for the existence of a (formal) recursion operator and, ultimately, for an infinite hierarchy of symmetries of a difference equation.

We present the recursion operators for the Viallet equation and all of the ABS equations in Section 4. These recursion operators generate infinite hierarchies of symmetries for every ABS equation. The corresponding canonical conservation laws provide us with an infinite sequence of densities and corresponding fluxes of increasing order. The very first term in this sequence was found by Hydon and Rasin for all of the ABS equations by a direct computation [22. It has been shown recently that the Gardner method, originally developed for integrable partial differential equations, can be adapted to difference equations and it was applied for the production of infinitely many conservation laws for $\mathrm{H} 1$ and some other equations, including non-symmetrical ones [23, 24].

Our work was partially inspired by the results obtained by Levi and Yamilov. In [25] they started to develop a theory of integrable difference equations based on the study of their symmetries. The goal of Levi and Yamilov theory is a classification of difference equations possessing symmetries. They made a good progress in a formalisation of the theory and they also discussed the issue of dynamical variables. Levi and Yamilov have found one integrability condition for difference equations and it does coincide with the first element of our infinite sequence of canonical 
conservation laws. Their approach is based solely on symmetries and does not use the concept of (formal) recursion operator.

We would like to stress that our assumption, that the difference equation is an affine-linear polynomial and its symmetries are some rational functions, has been made mainly for the clarity of presentation, and it is suitable for the ABS equations. This assumption can be removed under some inessential technical conditions, which guarantee that the difference equation has a unique solution for an open set of initial data. Obviously all results in this paper are valid for local analytic functions of the lattice variables.

\section{Basic definitions}

In this section, we introduce the elimination map $\mathcal{E}$ and define dynamical variables for quadrilateral affine-linear difference equations. To make the paper self-contained, we also give the basic definitions of symmetries and conservation laws of difference equations.

\subsection{Difference equations and dynamical variables}

Difference equations on $\mathbb{Z}^{2}$ can be seen as a discrete analog of partial differential equations. Let us denote by $u=u(n, m)$ a complex-valued function $u: \mathbb{Z}^{2} \mapsto \mathbb{C}$ where $n$ and $m$ are "independent variables" and $u$ will play the rôle of a "dependent" variable in a difference equation. Instead of partial derivatives we have two commuting shift maps $\mathcal{S}$ and $\mathcal{T}$ defined as

$\mathcal{S}: u \mapsto u_{1,0}=u(n+1, m), \quad \mathcal{T}: u \mapsto u_{0,1}=u(n, m+1), \quad \mathcal{S}^{p} \mathcal{T}^{q}: u \mapsto u_{p, q}=u(n+p, m+q)$.

For uniformity of the notation, it is convenient to denote the "unshifted" function $u$ as $u_{0,0}$. In the theory of difference equations we shall treat $u_{p, q}$ as variables. The set of all shifts of the variable $u$ will be denoted by $U=\left\{u_{p, q} \mid(p, q) \in \mathbb{Z}^{2}\right\}$. For a function $f=f\left(u_{p_{1}, q_{1}}, \ldots, u_{p_{k}, q_{k}}\right)$ of variables $u_{p, q}$ the action of the operators $\mathcal{S}, \mathcal{T}$ is defined as

$$
\mathcal{S}^{i} \mathcal{T}^{j}(f)=f_{i, j}=f\left(u_{p_{1}+i, q_{1}+j}, \ldots, u_{p_{k}+i, q_{k}+j}\right) .
$$

A quadrilateral difference equation can be defined as

$$
Q\left(u_{0,0}, u_{1,0}, u_{0,1}, u_{1,1}\right)=0
$$

where $Q\left(u_{0,0}, u_{1,0}, u_{0,1}, u_{1,1}\right)$ is an irreducible polynomial of the "dependent variable" $u=u_{0,0}$ and its shifts. Irreducibility means that $Q$ cannot be factorised and presented as a product of two polynomials. It is assumed that equation (2) is valid at every point $(n, m) \in \mathbb{Z}^{2}$ and thus (2) represents the infinite set of equations

$$
Q_{p, q}=Q\left(u_{p, q}, u_{p+1, q}, u_{p, q+1}, u_{p+1, q+1}\right)=0, \quad(p, q) \in \mathbb{Z}^{2} .
$$

In this paper we shall consider only polynomials $Q$. This restriction is not very essential for our construction, but allows us to make the presentation more transparent. Moreover, for quadrilateral equations we shall assume that $Q$ is an irreducible affine-linear polynomial which depends nontrivially on all variables, i.e.

$$
\frac{\partial Q}{\partial u_{i, j}} \neq 0, \quad \frac{\partial^{2} Q}{\partial^{2} u_{i, j}}=0, \quad i, j \in\{0,1\}, Q \in \mathbb{C}\left[u_{0,0}, u_{1,0}, u_{0,1}, u_{1,1}\right] .
$$


This is true for all of the ABS equations (except the trivial case of the H1 equation when $\alpha=\beta$ ).

Let $\mathbb{C}[U]$ be the ring of polynomials of the variables $U$, where it is assumed that every polynomial has a finite number of terms. Maps $\mathcal{S}$ and $\mathcal{T}$ are automorphisms of $\mathbb{C}[U]$ and thus $\mathbb{C}[U]$ is a difference ring. We denote $J_{Q}=\left\langle\left\{Q_{p, q}\right\}\right\rangle$ the ideal generated by the difference equation and all its shifts (3). Since $Q$ is an irreducible affine linear polynomial, the ideal $J_{Q}$ is prime and radical. For any $a \in J_{Q}$ we have $\mathcal{S}(a) \in J_{Q}$ and $\mathcal{T}(a) \in J_{Q}$. Thus $J_{Q}$ is a difference ideal and $\mathbb{C}[U] / J_{Q}$ is a difference quotient ring.

The fields of rational functions of variables

$$
U_{\mathbf{s}}=\left\{u_{n, 0} \mid n \in \mathbb{Z}\right\}, \quad U_{\mathbf{t}}=\left\{u_{0, n} \mid n \in \mathbb{Z}\right\}, \quad U_{0}=U_{\mathbf{s}} \cup U_{\mathbf{t}}
$$

are denoted as

$$
\mathcal{F}_{\mathbf{s}}=\mathbb{C}\left(U_{\mathbf{s}}\right), \quad \mathcal{F}_{\mathbf{t}}=\mathbb{C}\left(U_{\mathbf{t}}\right), \quad \mathcal{F}_{0}=\mathbb{C}\left(U_{0}\right)
$$

respectively. Rational functions of variables $u_{p, q}$ that can be restricted to the solutions of the difference equation (i.e. the denominators do not vanish on the solutions) form a field

$$
\mathcal{F}_{Q}=\left\{[a] /[b] \mid a, b \in \mathbb{C}[U], b \notin J_{Q}\right\},
$$

where $[a]$ denotes the class of equivalent polynomials (two polynomials $f, g \in \mathbb{C}[U]$ are equivalent, denoted by $f \equiv g$, if $\left.f-g \in J_{Q}\right)$. For $a, b, c, d \in \mathbb{C}[U], b, d \notin J_{Q}$, the two rational functions $a / b$ and $c / d$ represent the same element of $\mathcal{F}_{Q}$ if $a d-b c \in J_{Q}$. Such rational functions we shall call equivalent and denote $a / b \equiv c / d$. The values of equivalent rational functions coincide for all solutions of the difference equation.

In general, it is quite a tedious computational problem to work with polynomials and rational functions modulo an ideal. In our case the ideal $J_{Q}$ is generated by affine-linear polynomials $Q_{p, q}$ and we can uniquely resolve equation $Q=0$ with respect to each variable

$$
\begin{array}{ll}
u_{0,0}=F\left(u_{1,0}, u_{0,1}, u_{1,1}\right), & u_{1,0}=G\left(u_{0,0}, u_{0,1}, u_{1,1}\right), \\
u_{0,1}=H\left(u_{0,0}, u_{1,0}, u_{1,1}\right), & u_{1,1}=M\left(u_{0,0}, u_{1,0}, u_{0,1}\right) .
\end{array}
$$

Since $Q$ is an affine linear polynomial, functions $F, G, H$ and $M$ are rational functions of their arguments. Equations (44) enable us recursively and uniquely to express any variable $u_{p, q}$ in terms of the variables $U_{0}=U_{\mathbf{s}} \cup U_{\mathbf{t}}$.

Definition 1. For elements of $U$ the elimination map $\mathcal{E}: U \mapsto \mathbb{C}\left(U_{0}\right)$ is defined recursively:

$$
\begin{array}{ll}
\forall p \in \mathbb{Z}, & \mathcal{E}\left(u_{0, p}\right)=u_{0, p}, \quad \mathcal{E}\left(u_{p, 0}\right)=u_{p, 0}, \\
\text { if } p>0, q>0, & \mathcal{E}\left(u_{p, q}\right)=M\left(\mathcal{E}\left(u_{p-1, q-1}\right), \mathcal{E}\left(u_{p, q-1}\right), \mathcal{E}\left(u_{p-1, q}\right)\right), \\
\text { if } p<0, q>0, & \mathcal{E}\left(u_{p, q}\right)=H\left(\mathcal{E}\left(u_{p, q-1}\right), \mathcal{E}\left(u_{p+1, q-1}\right), \mathcal{E}\left(u_{p+1, q}\right)\right), \\
\text { if } p>0, q<0, & \mathcal{E}\left(u_{p, q}\right)=G\left(\mathcal{E}\left(u_{p-1, q}\right), \mathcal{E}\left(u_{p-1, q+1}\right), \mathcal{E}\left(u_{p, q+1}\right)\right), \\
\text { if } p<0, q<0, & \mathcal{E}\left(u_{p, q}\right)=F\left(\mathcal{E}\left(u_{p+1, q}\right), \mathcal{E}\left(u_{p, q+1}\right), \mathcal{E}\left(u_{p+1, q+1}\right)\right) .
\end{array}
$$

For polynomials $f\left(u_{p_{1}, q_{1}}, \ldots, u_{p_{k}, q_{k}}\right) \in \mathbb{C}[U]$ the elimination map $\mathcal{E}: \mathbb{C}[U] \mapsto \mathbb{C}\left(U_{0}\right)$ is defined as

$$
\mathcal{E}: f\left(u_{p_{1}, q_{1}}, \ldots, u_{p_{k}, q_{k}}\right) \mapsto f\left(\mathcal{E}\left(u_{p_{1}, q_{1}}\right), \ldots, \mathcal{E}\left(u_{p_{k}, q_{k}}\right)\right) \in \mathbb{C}\left(U_{0}\right) .
$$

For rational functions $a / b, a, b \in \mathbb{C}[U], b \notin J_{Q}$ the elimination map $\mathcal{E}$ is defined as

$$
\mathcal{E}: a / b \mapsto \mathcal{E}(a) / \mathcal{E}(b) .
$$


Variables $U_{0}$ we shall call the dynamical variables. it is easy to see that $\mathcal{E}\left(u_{p, q}\right)$ is a rational function of $|p|+|q|+1$ dynamical variables

$$
\mathcal{E}\left(u_{p, q}\right) \in \mathbb{C}\left(\left\{u_{n, 0}, u_{0, m}|0 \leq| n-p|\leq| p|, 0 \leq| m-q|\leq| q \mid\right\}\right) .
$$

The choice of the set of dynamical variables is not unique. Another popular choice is the so-called "staircase" set $\left\{u_{n, n}, u_{n, n+1} \mid n \in \mathbb{Z}\right\}$ which will not be used in this paper.

The elimination map $\mathcal{E}: \mathbb{C}[U] \mapsto \mathbb{C}\left(U_{0}\right)$ is a difference ring homomorphism, its kernel is $J_{Q}$, and its image is a difference ring isomorphic to the factor ring $\mathbb{C}[U] / J_{Q}$. The field $\mathbb{C}\left(U_{0}\right)$ is a difference field with automorphisms $\mathcal{E} \circ \mathcal{S}$ and $\mathcal{E} \circ \mathcal{T}$. The elimination map $\mathcal{E}: \mathcal{F}_{Q} \mapsto \mathbb{C}\left(U_{0}\right)$ is a difference field isomorphism. It is a useful tool to establish whether two rational functions $f, g$ of variables $U$ are equivalent (i.e. represent the same element of $\left.\mathcal{F}_{Q}\right): f \equiv g$ if and only if $\mathcal{E}(f)=\mathcal{E}(g)$. Later on we shall use logarithms of rational functions. The action of $\mathcal{E}$ on $\ln (a), a \in \mathcal{F}_{Q}, a \notin J_{Q}$ is defined as $\mathcal{E}(\ln (a))=\ln (\mathcal{E}(a))$ and thus we shall say that $\ln (a) \equiv \ln (b)$ if and only if $\ln (\mathcal{E}(a))=\ln (\mathcal{E}(b))$.

To illustrate the above construction, let us consider the difference equation $Q=0$ with

$$
Q=\left(u_{0,0}-u_{1,1}\right)\left(u_{1,0}-u_{0,1}\right)-\alpha+\beta, \quad \alpha, \beta \in \mathbb{C},
$$

which is known as the $\mathrm{H} 1$ equation in the ABS classification or the discrete potential KdV equation. First we should assume that $\alpha \neq \beta$, otherwise the polynomial $Q$ is reducible. It follows from Definition 1 that

$$
\begin{aligned}
& \mathcal{E}\left(u_{1,1}\right)=M\left(u_{0,0}, u_{1,0}, u_{0,1}\right)=u_{0,0}-\frac{\alpha-\beta}{u_{1,0}-u_{0,1}}, \\
& \mathcal{E}\left(u_{1,2}\right)=\frac{u_{0,1}^{2} u_{0,2}-u_{0,0} u_{0,1}^{2}-(\alpha-\beta) u_{1,0}+u_{0,0} u_{0,1} u_{1,0}-u_{0,1} u_{0,2} u_{1,0}}{\beta-\alpha-u_{0,0} u_{0,1}+u_{0,1} u_{0,2}+u_{0,0} u_{1,0}-u_{0,2} u_{1,0}}, \ldots .
\end{aligned}
$$

Two rational functions

$$
f=\frac{u_{0,0}-u_{1,1}}{u_{1,0}-u_{-1,0}}, \quad g=\frac{\alpha-\beta}{\left(u_{1,0}-u_{-1,0}\right)\left(u_{1,0}-u_{0,1}\right)}
$$

are equivalent $f \equiv g$ since

$$
\frac{u_{0,0}-u_{1,1}}{u_{1,0}-u_{-1,0}}-\frac{\alpha-\beta}{\left(u_{1,0}-u_{-1,0}\right)\left(u_{1,0}-u_{0,1}\right)}=\frac{Q}{\left(u_{1,0}-u_{-1,0}\right)\left(u_{1,0}-u_{0,1}\right)}
$$

and it is easy to check that $\mathcal{E}(f)=\mathcal{E}(g)=g$.

\subsection{Symmetries and conservation laws of difference equations}

Definition 2. Let $Q=0$ be a difference equation. Then $K \in \mathcal{F}_{Q}$ is called a generator of an infinitesimal symmetry (or simply, a symmetry) of the difference equation if

$$
D_{Q}(K) \equiv 0 .
$$

Here $D_{Q}$ is the Frechét derivative of $Q$ defined as

$$
D_{Q}=\sum_{i, j} Q_{u_{i, j}} \mathcal{S}^{i} \mathcal{T}^{j}, \quad Q_{u_{i, j}}=\frac{\partial Q}{\partial u_{i, j}}
$$


The sum in (9) is finite for a given $Q$. For a quadrilateral equation it has only four terms. Here $D_{Q}(K)$ is equal to zero as element of $\mathcal{F}_{Q}$, i.e. $D_{Q}(K) \subset J_{Q}$. The way to check it is to apply the elimination map: thus, $K$ is a symmetry if $\mathcal{E}\left(D_{Q}(K)\right)=0$.

If $K$ is a symmetry and $u=u(n, m)$ is a solution of a difference equation (2), then there is an infinitesimal transformation of solution $u$ :

$$
\hat{u}=u+\epsilon K
$$

satisfying

$$
Q\left(\hat{u}_{0,0}, \hat{u}_{1,0}, \hat{u}_{0,1}, \hat{u}_{1,1}\right) \equiv \mathcal{O}\left(\epsilon^{2}\right) .
$$

If the difference equation $Q=0$ admits symmetries, then they form a Lie algebra. Indeed, with a symmetry $K \in \mathcal{F}_{Q}$ we can associate an evolutionary derivation of $\mathcal{F}_{Q}$ (or a vector field on $\mathcal{F}_{Q}$ ):

$$
X_{K}=\sum_{(p, q) \in \mathbb{Z}^{2}} K_{p, q} \frac{\partial}{\partial u_{p, q}},
$$

where $K_{p, q}=\mathcal{S}^{p} \mathcal{T}^{q}(K)$. A derivation (a vector field) $X_{K}$ is called evolutionary if it commutes with the shift automorphisms: $\mathcal{S} X_{K}=X_{K} \mathcal{S}, \mathcal{T} X_{K}=X_{K} \mathcal{T}$. It follows from (8) that for any $a \in J_{Q}$ we have $X_{K}(a) \in J_{Q}$ and thus the evolutionary derivation $X_{K}$ is defined correctly on the difference field of fractions $\mathcal{F}_{Q}$.

If the difference equation admits two symmetries $F$ and $G$, then the commutator of the corresponding evolutionary derivations is an evolutionary derivation of $\mathcal{F}_{Q}$

$$
X_{F} X_{G}-X_{G} X_{F}=X_{H},
$$

where $H=[F, G]$, with $[F, G]$ denoting the Lie bracket

$$
[F, G]=X_{F}(G)-X_{G}(F)=D_{G}(F)-D_{F}(G) \in \mathcal{F}_{Q} .
$$

It is easy to see that $H$ is a symmetry, indeed

$$
D_{Q}(H)=X_{H}(Q)=X_{F} X_{G}(Q)-X_{G} X_{F}(Q)=X_{F} D_{Q}(G)-X_{G} D_{Q}(F) \equiv 0 .
$$

Symmetries form a linear space over $\mathbb{C}$. The bracket (10) is linear with respect to each argument, it is skew-symmetric and satisfies the Jacobi identity. The Lie algebra of symmetries of the difference equation $Q=0$ will be denoted as $\mathfrak{A}_{Q}$. Existence of an infinite dimensional Lie algebra $\mathfrak{A}_{Q}$ is a characteristic property of integrable equations and can be taken as a definition of integrability.

We would like to emphasise that a symmetry $K$ is an element of $\mathcal{F}_{Q}$, so it can be represented by a function $\mathcal{E}(K) \in \mathcal{F}_{0}$ of variables $U_{0}$. As an element of $\mathcal{F}_{0}$, a symmetry $K$ depends on a finite set of variables $\left\{u_{n, 0} \mid N_{1} \leq n \leq N_{2}\right\}$ and $\left\{u_{0, m} \mid M_{1} \leq n \leq M_{2}\right\}$, and thus can be characterised by four integers $\left(N_{1}, N_{2}, M_{1}, M_{2}\right)$. It is straightforward to check that, if

$$
K=K\left(u_{N_{1}, 0}, \ldots, u_{N_{2}, 0}, u_{0, M_{1}}, \ldots, u_{0, M_{2}}\right)
$$

is a symmetry of a quadrilateral equation, then it is a sum of two functions

$$
K=K_{\mathbf{s}}\left(u_{N_{1}, 0}, \ldots, u_{N_{2}, 0}\right)+K_{\mathbf{t}}\left(u_{0, M_{1}}, \ldots, u_{0, M_{2}}\right) .
$$


In particular, for the ABS equations it is known [21, 26] that, any "five point symmetry" $K=$ $K\left(u_{0,0}, u_{-1,0}, u_{0,-1}, u_{1,0}, u_{0,1}\right)$ is a sum of two symmetries $K=K_{\mathbf{s}}\left(u_{-1,0}, u_{0,0}, u_{1,0}\right)+K_{\mathbf{t}}\left(u_{0,-1}, u_{0,0}, u_{0,1}\right)$. Thus, the problem of the symmetry description is likely to be reduced to the study of the symmetries depending on "one-dimensional" set of variables $U_{\mathbf{s}}$ or $U_{\mathbf{t}}$. Moreover, all ABS equations and the Viallet equation possess infinite hierarchy of such symmetries.

Let $f=f\left(u_{N_{1}, 0}, \ldots, u_{N_{2}, 0}\right) \in \mathcal{F}_{\mathbf{s}}$ and $\partial f / \partial u_{N_{1}, 0} \neq 0, \partial f / \partial u_{N_{2}, 0} \neq 0$, then the order of $f$ is defined as $\operatorname{ord}_{\mathbf{s}}(f)=\left(N_{1}, N_{2}\right)$. For example,

$$
K^{(1)}=\left(u_{1,0}-u_{-1,0}\right)^{-1}
$$

is the generator of a symmetry for equation (7) and $\operatorname{ord}_{\mathbf{s}}\left(K^{(1)}\right)=(-1,1)$. Similarly, the order of $g=g\left(u_{0, M_{1}}, \ldots, u_{0, M_{2}}\right) \in \mathcal{F}_{\mathbf{t}}$ is defined as $\operatorname{ord}_{\mathbf{t}}(g)=\left(M_{1}, M_{2}\right)$.

Definition 3. 1. A pair of functions $\rho, \sigma \in \mathcal{F}_{Q}$ is called a conservation law for the difference equation (2), if

$$
(\mathcal{T}-\mathbf{1})(\rho) \equiv(\mathcal{S}-\mathbf{1})(\sigma)
$$

Functions $\rho$ and $\sigma$ will be referred to as the density and the flux of the conservation law and 1 denotes the identity map.

2. A conservation law is called trivial, if functions $\rho$ and $\sigma$ are components of a (difference) gradient of some element $H \in \mathcal{F}_{Q}$, i.e.

$$
\rho=(\mathcal{S}-\mathbf{1})(H), \quad \sigma=(\mathcal{T}-\mathbf{1})(H) .
$$

3. A non-constant element $C \in \mathcal{F}_{Q}$ is called $\mathbf{s}$-constant, (respectively $\mathbf{t}$-constant) if $\mathcal{S}(C)=C$ (respectively $\mathcal{T}(C)=C$ ). Elements of $\mathcal{F}_{Q}$ which are invariant with respect to the both shifts are called constants of $\mathcal{F}_{Q}$.

Existence of non-trivial conservation laws and/or $\mathbf{s}$ or $\mathbf{t}-$-constants for a difference equation is a rather exceptional property. The ABS equations (and many other integrable difference equations) possess infinitely many nontrivial conservation laws. Similar to the differential case [27, the existence of $\mathbf{s}$ or $\mathbf{t}$-constants suggests that the difference equation is linearizable. For instance the linear difference equation with $Q=u_{0,0}-u_{1,0}-u_{0,1}+u_{1,1}$ has a t-constant $f\left(u_{1,0}-u_{0,0}\right)$ and a s-constant $g\left(u_{0,1}-u_{0,0}\right)$, where $f, g$ are arbitrary functions.

Definition 4. Let $\operatorname{Im}(\mathcal{S}-\mathbf{1})$ and $\operatorname{Im}(\mathcal{T}-\mathbf{1})$ denote the images of the maps $\mathcal{S}-\mathbf{1}: \mathcal{F}_{Q} \mapsto \mathcal{F}_{Q}$ and $\mathcal{T}-\mathbf{1}: \mathcal{F}_{Q} \mapsto \mathcal{F}_{Q}$ respectively.

1. Two elements $\rho, \varrho \in \mathcal{F}_{Q}$ are $\mathbf{s}$-equivalent $\left(\rho \cong_{\mathbf{s}} \varrho\right)$ if $\rho-\varrho \in \operatorname{Im}(\mathcal{S}-\mathbf{1})$. Elements of the factor space $\mathcal{F}_{Q} / \operatorname{Im}(\mathcal{S}-\mathbf{1})$ we shall call $\mathbf{s}$-functionals, or densities.

2. Two elements $\sigma, \varsigma \in \mathcal{F}_{Q}$ are $\mathbf{t}$-equivalent $\left(\sigma \cong_{\mathbf{t}} \varsigma\right)$ if $\sigma-\varsigma \in \operatorname{Im}(\mathcal{T}-\mathbf{1})$. Elements of the factor space $\mathcal{F}_{Q} / \operatorname{Im}(\mathcal{T}-\mathbf{1})$ we shall call $\mathbf{t}$-functionals, or fluxes.

In general the problem to establish whether two elements of $\mathcal{F}_{Q}$ are $\mathbf{s}$ - or $\mathbf{t}$-equivalent is difficult. This problem can be easily solved for densities belonging to $\mathcal{F}_{\mathbf{s}}$ (or fluxes belonging to $\mathcal{F}_{\mathbf{t}}$ ). Moreover, in the next Section we will show that an integrable quadrilateral equation (such as the the Viallet equation) possesses an infinite hierarchies of canonical conservation laws such that their densities are elements of $\mathcal{F}_{\mathbf{s}}$.

There is a criterion to determine whether two elements of $\mathcal{F}_{\mathbf{S}}$ are equivalent or not. This is based on the notion of variational derivative (Euler's operator). 
Definition 5. Let $f \in \mathcal{F}_{\mathbf{s}}$ has order $\left(N_{1}, N_{2}\right)$, then the variational derivative $\delta_{\mathbf{s}}$ of $f$ is defined as

$$
\delta_{\mathbf{s}}(f)=\sum_{k=N_{1}}^{N_{2}} \mathcal{S}^{-k}\left(\frac{\partial f}{\partial u_{k, 0}}\right) .
$$

Two elements $\rho, \varrho \in \mathcal{F}_{\mathbf{s}}$ are equivalent $\rho \cong_{\mathbf{s}} \varrho$ if and only if $\delta_{\mathbf{s}}(\rho)=\delta_{\mathbf{s}}(\varrho)$. In particularly, if density $\rho \in \mathcal{F}_{\mathbf{s}}$ is trivial, then $\delta_{\mathbf{s}}(\rho)=0$. The order of a density $\rho \in \mathcal{F}_{\mathbf{s}}$ is defined as $\operatorname{ord}_{\delta_{\mathbf{s}}}(\rho)=$ $N_{2}-N_{1}$, where $\left(N_{1}, N_{2}\right)=\operatorname{ord}\left(\delta_{\mathbf{s}}(\rho)\right)$. Equivalent densities have the same order. For example the densities of conservation laws for the $\mathrm{H} 1$ equation (7)

$$
\rho_{0}=\ln \left(u_{1,0}-u_{-1,0}\right) \quad \text { and } \quad \rho_{1}=\frac{1}{\left(u_{1,0}-u_{-1,0}\right)\left(u_{2,0}-u_{0,0}\right)},
$$

are of orders 4 and 6 respectively.

\section{Recursion operator and canonical conservation laws}

In this section we give definitions of (s- and $\mathbf{t}-$ ) difference and pseudo-difference operators. We adapt the concept of recursion operators to difference equations and give the criteria for a pseudodifference operator to be a recursion operator of a quadrilateral difference equation. Using Laurent formal series and their residues we derive infinitely many integrability conditions for quadrilateral difference equations.

\subsection{Recursion operators of difference equations}

In order to proceed we need to define $\mathbf{s}$ - and $\mathbf{t}$-pseudo-difference operators.

Definition 6. (1) A s-difference operator $B$ of order $N$ with coefficients in $\mathcal{F}_{Q}$ is a finite sum of the form

$$
B=b_{N} \mathcal{S}^{N}+b_{N-1} \mathcal{S}^{N-1}+\cdots+b_{M} \mathcal{S}^{M}, \quad b_{k} \in \mathcal{F}_{Q}, \quad M \leq N, \quad N, M \in \mathbb{Z} .
$$

(2) An operator $L$ is called a $\mathbf{s - p s e u d o - d i f f e r e n c e ~ o p e r a t o r ~ i f ~ i t ~ i s ~ e i t h e r ~ a ~ f i n i t e ~ s u m ~ o f ~ t e r m s ~}$

$$
L=\sum B_{n} \circ C_{n}^{-1}
$$

(where $B_{n}$ and $C_{n}$ are $\mathbf{s}$-difference operators with coefficients in $\mathcal{F}_{Q}$ and $\circ$ denotes their composition) or a finite composition of $\mathbf{s}$-pseudo-difference operators of the form (15).

In the above definition we used the shift map $\mathcal{S}$, and thus we have defined the s-difference, s-pseudo-difference operators. Using the shift map $\mathcal{T}$ instead, one can define the t-difference and pseudo-difference operators. We shall omit $\mathbf{s -}$ (or $\mathbf{t}-$ ) and simply say difference or pseudo-difference operators if it is clear from the context.

There is a natural action of difference operators on all elements of $\mathcal{F}_{Q}$ but the action of pseudodifference operators is not generally defined. For a pseudo-difference operator $L$ (15) it can be defined on those elements of $a_{i} \in \mathcal{F}_{Q}$ that belong to the intersection of the image spaces of the difference operators $C_{n}$, so that the action of operators $C_{n}^{-1}\left(a_{i}\right)$ is defined, i.e. $C_{n}^{-1}\left(a_{i}\right) \in \mathcal{F}_{Q}$. A 
linear space that is a $\mathbb{C}$-span of all such elements $a_{i}$ is a domain of the pseudo-difference operator denoted by $\operatorname{Dom}(L)$.

By a recursion operator $\mathfrak{R}$ of a difference equation $Q=0$ we shall understand a s-pseudodifference operator such that

$$
\mathfrak{R}: \operatorname{Dom}(\mathfrak{R}) \cap \mathfrak{A}_{Q} \mapsto \mathfrak{A}_{Q},
$$

where $\mathfrak{A}_{Q}$ is the linear space of symmetries of this difference equation. In other words, if the action of $\mathfrak{R}$ on a symmetry $K \in \mathcal{F}_{0}$ is defined, i.e. $\mathfrak{R}(K) \in \mathcal{F}_{Q}$, then $\mathfrak{R}(K)$ is a symmetry of the same difference equation. Similarly, we define a t-pseudo-difference recursion operator $\hat{\mathfrak{R}}$ : $\operatorname{Dom}(\hat{\mathfrak{R}}) \cap \mathfrak{A}_{Q} \mapsto \mathfrak{A}_{Q}$. The operator of multiplication by a constant is a trivial recursion operator. In what follows we shall assume that $\mathfrak{R}, \hat{\mathfrak{R}}$ are nontrivial.

Theorem 1. Let $Q\left(u_{0,0}, u_{1,0}, u_{0,1}, u_{1,1}\right)=0$ be a difference equation.

(i) If there exist two $\mathbf{s}-$ pseudo-differential operators $\mathfrak{R}$ and $\mathfrak{P}$ such that

$$
D_{Q} \circ \mathfrak{R}=\mathfrak{P} \circ D_{Q},
$$

then $\mathfrak{R}$ is a recursion operator of the difference equation.

(ii) Relation (16) is valid if and only if

$$
\mathcal{T}(\mathfrak{R})-\mathfrak{R}=\left[\Phi \circ \mathfrak{R}, \Phi^{-1}\right],
$$

where $\Phi=\left(Q_{u_{1,1}} \mathcal{S}+Q_{u_{0,1}}\right)^{-1} \circ\left(Q_{u_{1,0}} \mathcal{S}+Q_{u_{0,0}}\right)$, and the operator $\mathfrak{P}$ satisfies

$$
\mathfrak{P}=\left(Q_{u_{1,0}} \mathcal{S}+Q_{u_{0,0}}\right) \circ \mathfrak{R} \circ\left(Q_{u_{1,0}} \mathcal{S}+Q_{u_{0,0}}\right)^{-1} .
$$

Proof. (i) If $K$ is a symmetry and $\mathfrak{R}(K) \in \mathcal{F}_{Q}$ it follows from (16) that $D_{Q} \mathfrak{R}(K)=\mathfrak{P} D_{Q}(K) \equiv 0$ and thus $\mathfrak{R}(K)$ is a symmetry.

To prove (ii), we represent $D_{Q}$ in the factorised form

$$
D_{Q}=Q_{u_{1,1}} \mathcal{S} \circ \mathcal{T}+Q_{u_{0,1}} \mathcal{T}+Q_{u_{1,0}} \mathcal{S}+Q_{u_{0,0}}=\left(Q_{u_{1,1}} \mathcal{S}+Q_{u_{0,1}}\right) \circ(\mathcal{T}+\Phi)
$$

where

$$
\Phi=\left(Q_{u_{1,1}} \mathcal{S}+Q_{u_{0,1}}\right)^{-1} \circ\left(Q_{u_{1,0}} \mathcal{S}+Q_{u_{0,0}}\right) .
$$

Then (16) can be rewritten as

$$
\mathcal{T}(\mathfrak{R}) \circ \mathcal{T}+\Phi \circ \mathfrak{R}=\left(Q_{u_{1,1}} \mathcal{S}+Q_{u_{0,1}}\right)^{-1} \circ \mathfrak{P} \circ\left(Q_{u_{1,1}} \mathcal{S}+Q_{u_{0,1}}\right) \circ(\mathcal{T}+\Phi),
$$

which is equivalent to

$$
\mathcal{T}(\mathfrak{R})=\left(Q_{u_{1,1}} \mathcal{S}+Q_{u_{0,1}}\right)^{-1} \circ \mathfrak{P} \circ\left(Q_{u_{1,1}} \mathcal{S}+Q_{u_{0,1}}\right)
$$

and

$$
\Phi \circ \mathfrak{R}=\left(Q_{u_{1,1}} \mathcal{S}+Q_{u_{0,1}}\right)^{-1} \circ \mathfrak{P} \circ\left(Q_{u_{1,1}} \mathcal{S}+Q_{u_{0,1}}\right) \circ \Phi .
$$

Using (19) we can rewrite (22) in the form (18). It follows from (21) and (22) that

$$
\Phi \circ \Re=\mathcal{T}(\mathfrak{R}) \circ \Phi
$$

The latter can be rewritten in the form (17).

In the same way one can prove the similar statement in the $\mathbf{t}$-direction. 
Theorem 2. Let $Q\left(u_{0,0}, u_{1,0}, u_{0,1}, u_{1,1}\right)=0$ be a difference equation.

(i) If there exist two $\mathbf{t}$-pseudo-differential operators $\hat{\mathfrak{R}}$ and $\hat{\mathfrak{P}}$ such that

$$
D_{Q} \circ \hat{\mathfrak{R}}=\hat{\mathfrak{P}} \circ D_{Q},
$$

then $\hat{\mathfrak{R}}$ is a recursion operator of the difference equation.

(ii) Relation (24) is valid if and only if

$$
\mathcal{S}(\hat{\mathfrak{R}})-\hat{\mathfrak{R}}=\left[\Psi \circ \hat{\mathfrak{R}}, \Psi^{-1}\right]
$$

where $\Psi=\left(Q_{u_{1,1}} \mathcal{T}+Q_{u_{1,0}}\right)^{-1} \circ\left(Q_{u_{0,1}} \mathcal{T}+Q_{u_{0,0}}\right)$, and the operator $\hat{\mathfrak{P}}$ can be written as

$$
\hat{\mathfrak{P}}=\left(Q_{u_{0,1}} \mathcal{T}+Q_{u_{0,0}}\right) \circ \hat{\mathfrak{R}} \circ\left(Q_{u_{0,1}} \mathcal{T}+Q_{u_{0,0}}\right)^{-1} .
$$

As in the case of partial differential equations, we show that if $\mathfrak{R}$ is a recursion operator, so is $\mathfrak{R}^{n}$ for all $n \in \mathbb{Z}$ in the following statement.

Corollary 1. 1. Under the conditions of (i) in Theorem 1 , the pseudo-difference operator $\mathfrak{R}$ satisfies the following equations

$$
\mathcal{T}\left(\mathfrak{R}^{n}\right)-\mathfrak{R}^{n}=\left[\Phi \circ \mathfrak{R}^{n}, \Phi^{-1}\right], \quad n \in \mathbb{Z},
$$

2. Under the conditions of (i) in Theorem 圆, the pseudo-difference operator $\hat{\mathfrak{R}}$ satisfies equations

$$
\mathcal{S}\left(\hat{\mathfrak{R}}^{n}\right)-\hat{\mathfrak{R}}^{n}=\left[\Psi \circ \hat{\mathfrak{R}}^{n}, \Psi^{-1}\right] \quad n \in \mathbb{Z},
$$

Proof. For the first part, it follows from (16) that $D_{Q} \circ \mathfrak{R}^{n}=\mathfrak{P}^{n} \circ D_{Q}, n \in \mathbb{Z}$. Thus, we can apply Theorem 1 to $\mathfrak{R}^{n}$ and $\mathfrak{P}^{n}$ to produce (26) from (17). The proof of the second part of the Corollary is similar.

In all our definitions and statements there is an obvious symmetry between " $\mathcal{T}$ " and " $\mathcal{S}$ " objects (such as symmetries, pseudo-differential operators, symplectic and Hamiltonian operators). In what follows we shall concentrate on "S" objects, but formulate some statements for both if required.

\subsection{Canonical conservation laws and integrability conditions}

Definition 7. A formal (Lauren 1 ) series of order $N$ is defined as a formal semi-infinite sum

$$
A=a_{N} \mathcal{S}^{N}+a_{N-1} \mathcal{S}^{N-1}+\cdots+a_{1} \mathcal{S}+a_{0}+a_{-1} \mathcal{S}^{-1}+\cdots, \quad a_{N} \neq 0, \quad a_{k} \in \mathcal{F}_{Q}, \quad N \in \mathbb{Z} .
$$

Laurent formal series form a skew-field. Sums and products (compositions) of formal series are formal series. The product is associative, but not commutative. For any formal series $A$ there exists a formal series $A^{-1}$ such that $A \circ A^{-1}=A^{-1} \circ A=1$. In order to find the first $n$ coefficients of $A^{-1}$ one needs to know exactly the first $n$ coefficients of $A$. The same holds for the

${ }^{1}$ One can also define a formal Taylor series of order $-N$ as a semi-infinite sum

$$
C=c_{-N} \mathcal{S}^{-N}+c_{1-N} \mathcal{S}^{1-N}+\cdots+c_{-1} \mathcal{S}^{-1}+c_{0}+c_{1} \mathcal{S}+\cdots, \quad c_{N} \neq 0, \quad c_{k} \in \mathcal{F}_{Q}, \quad N \in \mathbb{Z}
$$

to develop a similar theory based on the Taylor series (or both). But in the case when the recursion operator is a composition of a Hamiltonian and symplectic operators it can be shown that the both approaches give equivalent results. Thus in applications to the ABS and Viallet equations we restrict ourselves to Laurent formal series only. Study of the both (Laurent and Taylor) series make sense for asymmetric difference equations. 
composition of two formal series. For instance, for two Laurent formal series $A$ defined by (28), and $B=b_{M} \mathcal{S}^{M}+b_{M-1} \mathcal{S}^{M-1} \ldots$, we have

$$
A \circ B=\sum_{k=0}^{\infty} c_{N+M-k} \mathcal{S}^{N+M-k}, \quad c_{k}=\sum_{q=0}^{N+M-k} a_{N-q} \mathcal{S}^{N-q}\left(b_{q+k-N}\right) \in \mathcal{F}_{Q} .
$$

Any pseudo-difference operator $B$ can be uniquely represented by a Laurent formal series $B_{L}$. For example for $B=(a \mathcal{S}+b)^{-1}$ we have

$$
B_{L}=\alpha_{-1} \mathcal{S}^{-1}+\alpha_{-2} \mathcal{S}^{-2}+\alpha_{-3} \mathcal{S}^{-3}+\cdots
$$

where the coefficients $\alpha_{k} \in \mathcal{F}_{Q}$ can be found recursively:

$$
\alpha_{-1}=\mathcal{S}^{-1}\left(\frac{1}{a}\right), \alpha_{-n}=-\mathcal{S}^{-1}\left(\frac{\alpha_{1-n} b}{a}\right) .
$$

Definition 8. Let $A_{L}$ denote the Laurent series representations of a pseudo-differential operator A. Then $\operatorname{ord} A_{L}$ is called the Laurent order of $A$.

Now we introduce the residue of a formal series, as well as a discrete version of Adler's theorem [28], which will be used consequently to produce integrability conditions for quadrilateral difference equations.

Definition 9. Let $A$ defined by (28) be a formal series of order $N$. The residue $\operatorname{res}(A)$ and the logarithmic residue res $\ln (A)$ are defined as

$$
\operatorname{res}(A)=a_{0}, \quad \operatorname{res} \ln (A)=\ln \left(a_{N}\right) .
$$

We shall need the following difference analog of the Adler Theorem [28]:

Theorem 3. Let $A=a_{N} \mathcal{S}^{N}+a_{N-1} \mathcal{S}^{N-1} \ldots$ and $B=b_{M} \mathcal{S}^{M}+b_{M-1} \mathcal{S}^{M-1} \cdots$ be two Laurent formal series of order $N$ and $M$ respectively. Then

$$
\operatorname{res}[A, B]=(\mathcal{S}-\mathbf{1})(\sigma(A, B)),
$$

where

$$
\sigma(A, B)=\sum_{n=1}^{N} \sum_{k=1}^{n} \mathcal{S}^{-k}\left(a_{-n}\right) \mathcal{S}^{n-k}\left(b_{n}\right)-\sum_{n=1}^{M} \sum_{k=1}^{n} \mathcal{S}^{-k}\left(b_{-n}\right) \mathcal{S}^{n-k}\left(a_{n}\right) \in \mathcal{F}_{Q} .
$$

Proof. Only commutators of the form $\left[a_{p} \mathcal{S}^{p}, b_{-p} \mathcal{S}^{-p}\right], p \in \mathbb{Z}$ contribute to the residue. Assuming $p>0$, we get

$\left[a_{p} \mathcal{S}^{p}, b_{-p} \mathcal{S}^{-p}\right]=a_{p} \mathcal{S}^{p}\left(b_{-p}\right)-\mathcal{S}^{-p}\left(a_{p}\right) b_{-p}=\left(\mathcal{S}^{p}-\mathbf{1}\right)\left(\mathcal{S}^{-p}\left(a_{p}\right) b_{-p}\right)=(\mathcal{S}-\mathbf{1}) \sum_{k=1}^{p} \mathcal{S}^{p-k}\left(\mathcal{S}^{-p}\left(a_{p}\right) b_{-p}\right)$

For first order formal series $A$ the first $n$ coefficients of the series completely define the first $n$ residues $\rho_{0}=\operatorname{res} \ln A, \rho_{1}=\operatorname{res} A, \ldots, \rho^{n-1}=\operatorname{res} A^{n-1}$.

In the theory of pseudo-differential formal series (see for example [3, 5, 6]) for a series $A=$ $a D_{x}^{N}+b D_{x}^{N-1}+\cdots$ of order $N$ it is always possible to find the $N$-th root, i.e. a formal series 
$B=A^{\frac{1}{N}}=a^{\frac{1}{N}} D_{x}+\left(\frac{1}{N} a^{\frac{1}{N}-1} b-\frac{N-1}{N} a^{\frac{1}{N}-1} D_{x}(a)\right)+\cdots$ such that $B^{N}=A$ and, consequently, to parameterize the coefficients of $A$ by a sequence of canonical residues res $\ln B, \operatorname{res} B, \operatorname{res} B^{2}, \ldots$. In the case of formal difference series in general it is not possible to find the $N$-th root of a series $A=a_{N} \mathcal{S}^{N}+a_{N-1} \mathcal{S}^{N-1}+\cdots$, i.e. a first order series $B=\alpha_{1} \mathcal{S}+\alpha_{0}+\cdots$ with the coefficients in $\mathcal{F}_{Q}$, such that $B^{N}=A$. Indeed, computations show that to determine the coefficient $\alpha_{k}$ one needs to solve equations of the form

$$
\prod_{i=0}^{N-1} \mathcal{S}^{i}\left(\alpha_{1}\right)=a_{N}, \quad \alpha_{k}+\mathcal{S}\left(\alpha_{k}\right)+\cdots+\mathcal{S}^{N-1}\left(\alpha_{k}\right)=g_{k}, \quad k=0,-1,-2, \ldots
$$

for some $g_{k} \in \mathcal{F}_{Q}$. Obviously, a solution $\alpha_{k} \in \mathcal{F}_{Q}$ does not exist if $g_{k} \notin \operatorname{Im}\left(\mathbf{1}+\mathcal{S}+\cdots \mathcal{S}^{N-1}\right)$.

Theorem 4. If a difference equation possesses a recursion operator $\mathfrak{R}, \operatorname{ord}_{L}(\mathfrak{R})=N>0$, then it has infinitely many canonical conservation laws

$$
(\mathcal{T}-\mathbf{1}) \rho_{n N}=(\mathcal{S}-\mathbf{1}) \sigma_{n N}, \quad n=0,1,2, \ldots
$$

with canonical conserved densities

$$
\rho_{0}=\operatorname{res} \ln \mathfrak{R}_{L}, \quad \rho_{n N}=\operatorname{res} \mathfrak{R}_{L}^{n}, n>0 .
$$

Proof. Equation (26) is obviously satisfied if we replace $\Phi$ and $\mathfrak{R}$ by the corresponding Laurent formal series $\Phi_{L}$ and $\mathfrak{R}_{L}$. For $n \geq 1$, we compute the residue of equation (26) to arrive at

$$
\mathcal{T}\left(\operatorname{res} \Re_{L}^{n}\right)-\operatorname{res} \Re_{L}^{n}=\operatorname{res}\left(\left[\Phi_{L} \circ \mathfrak{R}_{L}^{n}, \quad \Phi_{L}^{-1}\right]\right)=(\mathcal{S}-\mathbf{1}) \sigma\left(\Phi_{L} \circ \mathfrak{R}_{L}^{n}, \Phi_{L}^{-1}\right) .
$$

It follows from Theorem 3 that

$$
\sigma_{n N}=\sigma\left(\Phi_{L} \circ \Re_{L}^{n}, \Phi_{L}^{-1}\right), \quad \sigma_{n} \in \mathcal{F}_{Q} .
$$

For $n=0$, we rewrite (23) as

$$
\Phi \circ \mathfrak{R} \circ \Phi^{-1} \circ \mathfrak{R}^{-1}=\mathcal{T}(\mathfrak{R}) \circ \mathfrak{R}^{-1} .
$$

and compute its logarithmic residue. This leads to

$$
\mathcal{T}\left(\operatorname{res} \ln \Re_{L}\right)-\operatorname{res} \ln \mathfrak{R}_{L}=(\mathcal{S}-\mathbf{1}) \sum_{k=0}^{N-1} \mathcal{S}^{k-1}\left(\ln \alpha_{0}\right),
$$

where $\alpha_{0}$ is the first coefficient in the expansion $\Phi_{L}=\alpha_{0}+\alpha_{1} \mathcal{S}^{-1}+\cdots$, (see (37))

In the above Theorem, densities and fluxes are counted modulo $N$, where $N$ is the Laurent order of the recursion operator.

If a recursion operator is known, then Theorem 4 gives us a completely algorithmic way to find explicitly a sequence of conservation laws, including both the densities $\rho_{k}$ and the corresponding fluxes $\sigma_{k}$. The residues of powers a formal series are easy to compute. For instance, if

$$
\mathfrak{R}=r_{1} \mathcal{S}+r_{0}+r_{-1} \mathcal{S}^{-1}+r_{-2} \mathcal{S}^{-2}+r_{-3} \mathcal{S}^{-3}+
$$

then

$$
\begin{aligned}
\operatorname{res} \ln \mathfrak{R}= & \ln r_{1}, \quad \operatorname{res} \mathfrak{R}=r_{0}, \quad \operatorname{res} \mathfrak{R}^{2}=\mathcal{S}^{-1}\left(r_{1}\right) r_{-1}+r_{0}^{2}+r_{1} \mathcal{S}^{-1}\left(r_{-1}\right) \\
\operatorname{res} \mathfrak{R}^{3}= & \mathcal{S}^{-2}\left(r_{1}\right) \mathcal{S}^{-1}\left(r_{1}\right) r_{-2}+\mathcal{S}^{-1}\left(r_{0}\right) \mathcal{S}^{-1}\left(r_{1}\right) r_{-1}+2 \mathcal{S}^{-1}\left(r_{1}\right) r_{-1} r_{0} \\
& +\mathcal{S}^{-1}\left(r_{1}\right) r_{1} \mathcal{S}\left(r_{-2}\right)+r_{0}^{3}+2 r_{0} r_{1} \mathcal{S}\left(r_{-2}\right)+r_{1} \mathcal{S}\left(r_{-1}\right) \mathcal{S}\left(r_{0}\right)+r_{1} \mathcal{S}\left(r_{1}\right) \mathcal{S}^{2}\left(r_{-2}\right) .
\end{aligned}
$$


Proposition 1. If a recursion operator $\mathfrak{R}$ is represented by a first order formal series $\mathfrak{R}_{L}=$ $r_{1} \mathcal{S}+r_{0}+r_{-1} \mathcal{S}^{-1}+\cdots$, then

$$
\begin{aligned}
& (\mathcal{T}-\mathbf{1})\left(\ln r_{1}\right)=(\mathcal{S}-\mathbf{1}) \mathcal{S}^{-1}\left(\ln \frac{Q_{u_{1,1}}}{Q_{u_{1,0}}}\right) \\
& (\mathcal{T}-\mathbf{1})\left(r_{0}\right)=(\mathcal{S}-\mathbf{1}) \mathcal{S}^{-1}\left(r_{1} F\right) \\
& (\mathcal{T}-\mathbf{1})\left(r_{-1} \mathcal{S}^{-1}\left(r_{1}\right)+r_{0}^{2}+r_{1} \mathcal{S}\left(r_{-1}\right)\right)=(\mathcal{S}-\mathbf{1})\left(\sigma_{2}\right),
\end{aligned}
$$

where

$$
\sigma_{2}=\mathcal{S}^{-1}\left(r_{1} F\right)\left\{\mathcal{S}^{-1}\left(r_{0}\right)+r_{0}-\mathcal{S}^{-2}\left(r_{1} F\right)\right\}-\left(1+\mathcal{S}^{-1}\right)\left(r_{1} G \mathcal{S}^{-1}\left(r_{1} F\right)\right),
$$

and $F, G$ denote

$$
F=\frac{Q_{u_{0,1}} \mathcal{S}^{-1}\left(Q_{u_{1,0}}\right)-Q_{u_{0,0}} \mathcal{S}^{-1}\left(Q_{u_{1,1}}\right)}{Q_{u_{1,0}} \mathcal{S}^{-1}\left(Q_{u_{1,1}}\right)}, \quad G=\frac{Q_{u_{0,0}}}{Q_{u_{1,0}}} .
$$

Proof. We expand $\Phi$ and $\Phi^{-1}(\underline{19})$ in the formal Laurent series

$$
\Phi_{L}=\alpha_{0}+\alpha_{1} \mathcal{S}^{-1}+\alpha_{2} \mathcal{S}^{-2}+\ldots, \quad \Phi_{L}^{-1}=\beta_{0}+\beta_{1} \mathcal{S}^{-1}+\beta_{2} \mathcal{S}^{-2}+\ldots .
$$

The coefficients $\alpha_{k}, \beta_{k} \in \mathcal{F}_{Q}$ can be found recursively:

$$
\begin{array}{ll}
\alpha_{0}=\mathcal{S}^{-1}\left(\frac{Q_{u_{1,0}}}{Q_{u_{1}, 1}}\right), & \beta_{0}=\mathcal{S}^{-1}\left(\frac{Q_{u_{1,1}}}{Q_{u_{1}, 0}}\right), \\
\alpha_{1}=\mathcal{S}^{-1}\left(\frac{Q_{u_{0}, 0}}{Q_{u_{1,1}}}-\frac{Q_{u_{0,1}}}{Q_{u_{1,1}}} \mathcal{S}^{-1}\left(\frac{Q_{u_{1,0}}}{Q_{u_{1,1}}}\right)\right), & \beta_{1}=\mathcal{S}^{-1}\left(\frac{Q_{u_{0}, 1}}{Q_{u_{1,0}}}-\frac{Q_{u_{0,0}}}{Q_{u_{1}, 0}} \mathcal{S}^{-1}\left(\frac{Q_{u_{1,1}}}{Q_{u_{1,0}}}\right)\right), \\
\alpha_{n+1}=(-1)^{n} \mathcal{S}^{-1}\left(\frac{Q_{u_{0,1}}}{Q_{u_{1,1}}} \alpha_{n}\right), & \beta_{n+1}=(-1)^{n} \mathcal{S}^{-1}\left(\frac{Q_{u_{0,0}}}{Q_{u_{1,0}}} \beta_{n}\right), \quad n \geq 1 .
\end{array}
$$

Then (34) follows from (32) and formulas (35) and (36) follow from (30) and (31) for $n=1$ and $n=2$ respectively.

There is no obstacle to compute equations corresponding to $n=3,4,5$, etc. Of course, the expressions get bigger. Obviously, if we consider the recursion operators $\hat{\Re}$ in the $\mathcal{T}$ direction, we immediately get :

Proposition 2. If a recursion operator $\hat{\mathfrak{R}}$ is represented by a first order formal Laurent $\mathcal{T}$ series $\hat{\mathfrak{R}}_{L}=\hat{r}_{1} \mathcal{T}+\hat{r}_{0}+\hat{r}_{-1} \mathcal{T}^{-1}+\cdots$, then

$$
\begin{array}{ll}
\text { (i) } & (\mathcal{S}-\mathbf{1})\left(\ln \hat{r}_{1}\right)=(\mathcal{T}-\mathbf{1}) \mathcal{T}^{-1}\left(\ln \frac{Q_{u_{1,1}}}{Q_{u_{0,1}}}\right), \\
\text { (ii) } & (\mathcal{S}-\mathbf{1})\left(\hat{r}_{0}\right)=(\mathcal{T}-\mathbf{1}) \mathcal{T}^{-1}\left(\hat{r}_{1} \hat{F}\right), \\
\text { (iii) } & (\mathcal{S}-\mathbf{1})\left(\hat{r}_{-1} \mathcal{S}^{-1}\left(\hat{r}_{1}\right)+\hat{r}_{0}^{2}+\hat{r}_{1} \mathcal{S}\left(\hat{r}_{-1}\right)\right)=(\mathcal{T}-\mathbf{1})\left(\hat{\sigma}_{2}\right),
\end{array}
$$

where

$$
\hat{\sigma}_{2}=\mathcal{S}^{-1}\left(\hat{r}_{1} \hat{F}\right)\left\{\mathcal{S}^{-1}\left(\hat{r}_{0}\right)+\hat{r}_{0}-\mathcal{S}^{-2}\left(\hat{r}_{1} \hat{F}\right)\right\}-\left(1+\mathcal{S}^{-1}\right)\left(\hat{r}_{1} \hat{G} \mathcal{S}^{-1}\left(\hat{r}_{1} \hat{F}\right)\right),
$$

and $\hat{F}, \hat{G}$ denote

$$
\hat{F}=\frac{Q_{u_{1,0}} \mathcal{T}^{-1}\left(Q_{u_{0,1}}\right)-Q_{u_{0,0}} \mathcal{T}^{-1}\left(Q_{u_{1,1}}\right)}{Q_{u_{0,1}} \mathcal{T}^{-1}\left(Q_{u_{1,1}}\right)}, \quad \hat{G}=\frac{Q_{u_{0,0}}}{Q_{u_{0,1}}}
$$


It is not difficult to show that the existence of a formal series satisfying equation (17) is the necessary condition for the existence of a hierarchy of higher symmetries (of increasing order) for a difference equation. Thus, formulas (34, 36) and (38 40) can be seen as integrability conditions for the difference equation defined by function $Q$. Indeed, the right hand sides of these formulas are expressed in the terms of the difference equation only, and it is a rather non-trivial fact that the corresponding left hand sides are in the image space of the operators $\mathcal{T}-\mathbf{1}$ or $\mathcal{S}-\mathbf{1}$ respectively. If this is the case we can find the coefficients $r_{1}, \hat{r}_{1}$ and proceed to the next conditions (35) and (39) and so on.

Theorem 4provides infinitely many integrability condition of that type. Integrability conditions (34) and (38) have been earlier found by Levi and Yamilov [25] from the symmetry analysis of difference equations. They actually found two more conditions of this type, which in our setup correspond to the Taylor formal series (see footnote 11) and can be easily recovered. Using the Taylor formal series we can double the number of the conditions, which would be appropriate for asymmetric or linearisable equations.

It is not a straightforward exercise to apply the integrability conditions obtained. Nevertheless, they can be perfectly used for testing integrability as well as for classification of integrable difference equations (see for example [25]).

\section{Recursion operator, symmetries and conservation laws for the Viallet equation}

In this section we apply the theory developed in the previous sections to the case of the Viallet equation, as well as to the ABS equations. The first is characterized as integrable because its algebraic entropy vanishes, [20], while the multidimensional consistency of latter implies their integrability, [17]. Here, we will show that they are integrable also in the sense that they admit infinite hierarchies of symmetries by constructing recursion operators for all of them. In order to make our presentation self-contained, we first introduce some properties of these equations. Next we present two symmetries and a recursion operator for the Viallet equation and demonstrate how it is related to corresponding operators for all of the ABS equations through an explicit example. Finally, we present some higher order conservation laws for all of the equations under consideration.

Throughout this section we assume that, the defining function $Q$ of equation (1) is irreducible. This means that parameters $a_{i}$ cannot take values for which $Q$ can be factorized as a product of two polynomials. By making appropriate choices of the coefficients $a_{i}$ we can reduce the Viallet equation to every equation from the ABS list (see Appendix). The ABS equations depend on two so-called lattice parameters $\alpha$ and $\beta$.

Let us introduce two polynomials in terms of $Q$ for equation (1), which will be used in the rest of this section. They are determined by the discriminants of $Q$,

$$
\begin{aligned}
& h\left(u_{0,0}, u_{1,0}\right):=Q \partial_{u_{0,1}} \partial_{u_{1,1}} Q-\partial_{u_{0,1}} Q \partial_{u_{1,1}} Q, \\
& \hat{h}\left(u_{0,0}, u_{0,1}\right):=Q \partial_{u_{1,0}} \partial_{u_{1,1}} Q-\partial_{u_{1,0}} Q \partial_{u_{1,1}} Q, .
\end{aligned}
$$

Polynomials $h\left(u_{0,0}, u_{1,0}\right), \hat{h}\left(u_{0,0}, u_{0,1}\right)$ are symmetric and biquadratic [18. In the case of the ABS equations the polynomial $h$ can be factorised and presented in the form

$$
h(u, x)=k(\alpha, \beta) f(u, x ; \alpha),
$$


where $k$ is a skew-symmetric function of the lattice parameters and $f$ is symmetric and biquadratic polynomial of $u$ and $x$ [17]. Polynomials $f(u, x ; \alpha)$ for the ABS equations are listed in the Appendix.

The covariance of these equations is another useful property for our presentation. For the Viallet equation this means that it is invariant under interchanging $u_{1,0}$ and $u_{0,1}$. For all of the ABS equations, covariance means that we have to interchange not only $u_{1,0}$ and $u_{0,1}$, but the lattice parameters $\alpha$ and $\beta$ as well. This property requires us to study symmetries, recursion operators and conservation laws only for the one direction of the lattice (and recover the complementary set using the covariance). We shall assume that symmetries and canonical densities are elements of $\mathcal{F}_{\mathbf{s}}$ and the recursion operator is a s-pseudo-difference operator. In most cases functions shifted by $\mathcal{S}$ only will contribute to the expressions. For this reason from now on, we will use one-index notation for the shifts of the polynomial $h$, i.e.

$$
h=h\left(u_{0,0}, u_{1,0}\right), \quad h_{i}=\mathcal{S}^{i} h\left(u_{0,0}, u_{1,0}\right),
$$

and symmetries, e.g. $K_{j}^{(1)}=\mathcal{S}^{j}\left(K^{(1)}\right)$. We shall omit the index zero for unshifted functions.

We now turn our attention to the symmetry analysis of the equations. It is known [21, 26] that these equations admit a generalised symmetry $K^{(1)}$ of order $(-1,1)$, which is given by the formula

$$
K^{(1)}:=\frac{h}{u_{1,0}-u_{-1,0}}-\frac{1}{2} \partial_{u_{1,0}} h=\frac{h_{-1}}{u_{1,0}-u_{-1,0}}+\frac{1}{2} \partial_{u_{-1,0}} h_{-1} .
$$

Using Definition 2 and formula (8), we can directly verify the following result:

Proposition 3. The Viallet equation (1) possesses a symmetry of order $(-2,2)$ which has the form

$$
K^{(2)}=\frac{h h_{-1}}{\left(u_{1,0}-u_{-1,0}\right)^{2}}\left(\frac{1}{u_{2,0}-u_{0,0}}+\frac{1}{u_{0,0}-u_{-2,0}}\right) .
$$

Actually, the Viallet equation possesses infinitely many local generalised symmetries. These hierarchies of symmetries can be constructed by applying successively a recursion operator $\mathfrak{R}$ on the seed or root symmetries $K^{(1)}$ and $K^{(2)}$, the starting points for a hierarchy of symmetries [29].

Theorem 5. The Viallet equation (1) possesses a recursion operator $\mathfrak{R}=\mathcal{H} \circ \mathcal{I}$, where operators $\mathcal{H}$ and $\mathcal{I}$ are defined as

$$
\begin{aligned}
\mathcal{I}= & \frac{1}{h} \mathcal{S}-\mathcal{S}^{-1} \frac{1}{h} ; \\
\mathcal{H}= & \frac{h_{-1} h h_{1}}{\left(u_{1,0}-u_{-1,0}\right)^{2}\left(u_{2,0}-u_{0,0}\right)^{2}} \mathcal{S}-\mathcal{S}^{-1} \frac{h_{-1} h h_{1}}{\left(u_{1,0}-u_{-1,0}\right)^{2}\left(u_{2,0}-u_{0,0}\right)^{2}} \\
& +2 K^{(1)} \mathcal{S}(\mathcal{S}-\mathbf{1})^{-1} \circ K^{(2)}+2 K^{(2)}(\mathcal{S}-\mathbf{1})^{-1} \circ K^{(1)} .
\end{aligned}
$$

In this Theorem $\mathcal{H}$ and $\mathcal{I}$ are compatible Hamiltonian and symplectic operators for the Viallet equation, they will be discussed in details in our paper 30. including a complete proof of the Theorem. In the same paper, we will also show that both $\mathfrak{R}^{\ell}\left(K^{(1)}\right)$ and $\mathfrak{R}^{\ell}\left(K^{(2)}\right)$ are in $\mathcal{F}_{\mathbf{s}}$ for all $\ell \in \mathbb{N}$. This is not obvious at all, since the Hamiltonian operator $\mathcal{H}$ contains a pseudo-difference operator $(\mathcal{S}-\mathbf{1})^{-1}$. Symmetries $K^{(1)}$ and $K^{(2)}$ are called the seed (or root) symmetries for the Viallet equation. Starting from them, two infinite hierarchies of symmetries can be generated by applying the operator $\mathfrak{R}^{\ell}$. Later in this Section we shall demonstrate the proof of Theorem 5 for the H1 equation (Theorem 6).

As an application, we now compute explicitly the action of $\mathfrak{R}$ on the first symmetry $K^{(1)}$. 
Proposition 4. The Viallet equation (1) possesses a symmetry of order $(-3,3)$ which is given by

$$
\begin{aligned}
K^{(3)}:=\mathfrak{R}\left(K^{(1)}\right)= & \frac{h h_{-1}}{\left(u_{1,0}-u_{-1,0}\right)^{2}}\left(\frac{K_{2}^{(1)}}{\left(u_{2,0}-u_{0,0}\right)^{2}}+\frac{K_{-2}^{(1)}}{\left(u_{0,0}-u_{-2,0}\right)^{2}}\right) \\
& +\left(\frac{1}{u_{2,0}-u_{0,0}}+\frac{1}{u_{0,0}-u_{-2,0}}\right) K^{(1)} K^{(2)} .
\end{aligned}
$$

Proof. To shorten the expression, we introduce the notation:

$$
w:=\frac{1}{u_{1,0}-u_{-1,0}}, \quad w_{k}=\mathcal{S}^{k}(w) .
$$

First we work out the product of operator $\mathcal{H}$ and $\mathcal{I}$

$$
\begin{aligned}
\mathfrak{R} & =h h_{-1} w^{2}\left(w_{1}^{2} \mathcal{S}^{2}+\mathcal{S}^{-2} w_{1}^{2}\right)+2 K^{(1)} K^{(2)}\left(\frac{1}{h} \mathcal{S}+\mathcal{S}^{-1} \frac{1}{h}\right) \\
& -w^{2}\left(h_{-1} h_{1} w_{1}^{2}+h_{-2} h w_{-1}^{2}\right)+\frac{2}{h_{-1}}\left(K^{(1)} K_{-1}^{(2)}+K^{(2)} K_{-1}^{(1)}\right) \\
& +2 K^{(1)}(\mathcal{S}-\mathbf{1})^{-1} \circ\left(\frac{K_{-1}^{(2)}}{h_{-1}}-\frac{K_{1}^{(2)}}{h}\right)+2 K^{(2)}(\mathcal{S}-\mathbf{1})^{-1} \circ\left(\frac{K_{-1}^{(1)}}{h_{-1}}-\frac{K_{1}^{(1)}}{h}\right) .
\end{aligned}
$$

Acting with operator $\mathfrak{R}$ on the symmetry $K^{(1)}$ and using the identities

$$
\begin{aligned}
K^{(i)} \mathcal{I} K^{(i)}= & (\mathcal{S}-\mathbf{1})\left(\frac{1}{h_{-1}} K^{(i)} K_{-1}^{(i)}\right), \quad i=1,2 ; \\
K^{(2)} \mathcal{I} K^{(1)}= & (\mathcal{S}-\mathbf{1})\left(\frac{-1}{h} K^{(2)} K_{1}^{(1)}+\frac{1}{2}\left(\frac{1}{u_{2,0}-u_{0,0}}+\frac{1}{u_{0,0}-u_{-2,0}}\right) K^{(2)}\right) \\
& +(\mathcal{S}-\mathbf{1})(\mathcal{S}+\mathbf{1})\left(\frac{h h_{-2}}{2\left(u_{1,0}-u_{-1,0}\right)^{2}\left(u_{0,0}-u_{-2,0}\right)^{2}}\right) \\
K^{(1)} \mathcal{I} K^{(2)}= & -K^{(2)} \mathcal{I} K^{(1)}+(\mathcal{S}-\mathbf{1})\left(\frac{1}{h_{-1}} K^{(1)} K_{-1}^{(2)}+\frac{1}{h_{-1}} K^{(2)} K_{-1}^{(1)}\right)
\end{aligned}
$$

we obtain expression (48) for symmetry $K^{(3)}$.

Using the above identities, one can easily compute the symmetry $K^{(4)}=\mathfrak{R}\left(K^{(2)}\right)$ of order $(-4,4)$. Applying $\mathfrak{R}^{N}$ to the seeds $K^{(1)}$ and $K^{(2)}$ we can generate two hierarchies of symmetries of order $(-2 N-1,2 N+1)$ and $(-2 N-2,2 N+2)$ respectively for the Viallet equation.

Remark 1. Symmetries and recursion operators for the ABS equations can be obtained from the symmetries of the Viallet equation and the operator $\mathfrak{R}=\mathcal{H} \mathcal{I}$, given in Theorem 5 , by replacing the polynomial $h$ (everywhere in the symmetries and the operators $\mathcal{I}, \mathcal{H}$ ) with the corresponding polynomials $f:=f\left(u_{0,0}, u_{1,0}, \alpha\right)$. For all $A B S$ equations the polynomials $f$ are listed in the Appendix. Equations $H 1-H 3, Q 1$ and $Q 3_{\delta=0}$ admit point symmetries [26]. For these equations, we find additional Hamiltonian operators of zero Laurent order which are given in the Appendix.

Proposition 5. If the recursion operator $\mathfrak{R}$, defined in Theorem 5 , is restricted to the H1 equation

$$
\left(u_{0,0}-u_{1,1}\right)\left(u_{1,0}-u_{0,1}\right)=\alpha-\beta,
$$


then it becomes

$$
\mathfrak{R}=\mathfrak{R}_{H 1}^{2}
$$

where

$$
\mathfrak{R}_{H 1}=\frac{1}{u_{1,0}-u_{-1,0}}(\mathcal{S}+\mathbf{1})(\mathcal{S}-\mathbf{1})^{-1} \frac{1}{u_{1,0}-u_{-1,0}}\left(\mathcal{S}-\mathcal{S}^{-1}\right) .
$$

Proof. The restriction to the H1 equation implies that $h=1$. Thus, the first two symmetries (44) and (45) become

$$
K^{(1)}=\frac{1}{u_{1,0}-u_{-1,0}} \quad \text { and } \quad K^{(2)}=\frac{1}{\left(u_{1,0}-u_{-1,0}\right)^{2}}\left(\frac{1}{u_{2,0}-u_{0,0}}+\frac{1}{u_{0,0}-u_{-2,0}}\right),
$$

respectively. Substituting them into (49), a direct calculation leads to the statement.

The operator given by (51) is a recursion operator of the $\mathrm{H} 1$ equation. To prove it, we will use the following result.

Lemma 1. Operator

$$
\begin{aligned}
\mathfrak{M}= & \frac{1}{\left(u_{2,1}-u_{0,1}\right)^{2}} \mathcal{S}+\frac{1}{\left(u_{1,0}-u_{-1,0}\right)^{2}} \mathcal{S}^{-1}+\frac{2}{\left(u_{1,1}-u_{-1,1}\right)\left(u_{2,1}-u_{0,1}\right)} \\
& +(\alpha-\beta)\left(\frac{1}{\left(u_{1,1}-u_{-1,1}\right)^{2}\left(u_{1,0}-u_{0,1}\right)^{2}}-\frac{1}{\left(u_{2,1}-u_{0,1}\right)^{2}\left(u_{2,0}-u_{1,1}\right)^{2}}\right) \\
& -2\left(\frac{1}{u_{1,0}-u_{-1,0}}+\frac{\alpha-\beta}{\left(u_{2,0}-u_{0,0}\right)\left(u_{1,0}-u_{0,1}\right)^{2}}\right) \times \\
& (\mathcal{S}-\mathbf{1})^{-1} \circ\left(\frac{1}{u_{2,0}-u_{0,0}}-\frac{u_{1,0}-u_{0,1}}{\left(u_{1,0}-u_{-1,0}\right)\left(u_{0,0}-u_{-1,1}\right)}\right)
\end{aligned}
$$

satisfies the following identity for all solutions of the H1 equation (50):

$$
\begin{aligned}
& \left(\mathbf{1}+\frac{\alpha-\beta}{\left(u_{1,0}-u_{0,1}\right)^{2}} \mathcal{S}\right) \circ \mathfrak{R}_{H 1}=\mathfrak{M} \circ\left(\mathbf{1}+\frac{\alpha-\beta}{\left(u_{1,0}-u_{0,1}\right)^{2}} \mathcal{S}\right) ; \\
& \left(\mathcal{S}+\frac{\alpha-\beta}{\left(u_{1,0}-u_{0,1}\right)^{2}}\right) \circ \mathcal{T}\left(\mathfrak{R}_{H 1}\right)=\mathfrak{M} \circ\left(\mathcal{S}+\frac{\alpha-\beta}{\left(u_{1,0}-u_{0,1}\right)^{2}}\right) .
\end{aligned}
$$

Proof. Writing out explicitly both sides of equation (53), we factorise terms in both sides according to the different powers of $\mathcal{S}$. Collecting the coefficients of the different powers of $\mathcal{S}$ in both sides of the resulting expression, their equality follows by putting them into $\mathcal{F}_{0}$. In the same way, we can prove identity (54).

Theorem 6. Operator $\mathfrak{R}_{H 1}$ given by (51) is a recursion operator of the H1 equation (50) with

$$
\mathfrak{P}=\left(u_{1,0}-u_{0,1}\right) \mathfrak{M} \circ\left(u_{1,0}-u_{0,1}\right)^{-1} .
$$

Proof. From Theorem 1 it follows that, $\mathfrak{R}_{H 1}$ is a recursion operator of the H1 equation provided both formulas (17) and (18) are valid for operator $\mathfrak{P}$. We rewrite them as

$$
\begin{aligned}
& \left(Q_{u_{1,0}} \mathcal{S}+Q_{u_{0,0}}\right) \circ \mathfrak{R}_{H 1}=\mathfrak{P} \circ\left(Q_{u_{1,0}} \mathcal{S}+Q_{u_{0,0}}\right) \\
& \left(Q_{u_{1,1}} \mathcal{S}+Q_{u_{0,1}}\right) \circ \mathcal{T}\left(\mathfrak{R}_{H 1}\right)=\mathfrak{P} \circ\left(Q_{u_{1,1}} \mathcal{S}+Q_{u_{0,1}}\right) .
\end{aligned}
$$


For the H1 equation, we have

$$
\begin{gathered}
Q_{u_{1,0}} \mathcal{S}+Q_{u_{0,0}}=\left(u_{0,0}-u_{1,1}\right) \mathcal{S}+\left(u_{1,0}-u_{0,1}\right)=\left(u_{1,0}-u_{0,1}\right)\left(\mathbf{1}+\frac{\alpha-\beta}{\left(u_{1,0}-u_{0,1}\right)^{2}} \mathcal{S}\right) \\
Q_{u_{1,1}} \mathcal{S}+Q_{u_{0,1}}=-\left(u_{1,0}-u_{0,1}\right) \mathcal{S}-\left(u_{0,0}-u_{1,1}\right)=-\left(u_{1,0}-u_{0,1}\right)\left(\mathcal{S}+\frac{\alpha-\beta}{\left(u_{1,0}-u_{0,1}\right)^{2}}\right) .
\end{gathered}
$$

Using Lemma 1, we can easily obtain (56) and (57) and thus we proved the statement.

Having constructed a recursion operator for equation (1), one can use the results of Section 3.2 to find conserved densities for this equation. If we apply directly Theorem 4 to the recursion operator (49), since the Laurent order of the recursion operator $\mathfrak{R}$ is two, we will get only the "even" part of the infinite sequence of the canonical conservation laws. Although it is impossible to compute a square root of a generic difference formal series, for the recursion operator (49) it can be done and thus one can find a first order formal recursion operator

$$
\tilde{\mathfrak{R}}=\frac{h_{-1}}{\left(u_{1,0}-u_{-1,0}\right)^{2}} \mathcal{S}+\frac{2 K^{(1)}}{u_{0,0}-u_{-2,0}}-\partial_{u_{0,0}} K^{(1)}+\cdots,
$$

such that $\tilde{\mathfrak{R}}^{2}=\mathfrak{R}$. The first order recursion operator provides us with a complete set of canonical conservation laws and the first three densities of this sequence are

$$
\begin{aligned}
\rho_{0} & =\ln \left(\partial_{u_{1,0}} K^{(1)}\right)=\ln h_{-1}-2 \ln \left(u_{1,0}-u_{-1,0}\right), \\
\rho_{1} & =\frac{2 K^{(1)}}{u_{0,0}-u_{-2,0}}-\partial_{u_{0,0}} K^{(1)}, \\
\rho_{2} & =\frac{-1}{\left(u_{1,0}-u_{-1,0}\right)^{2}}\left(\frac{h_{-1} h_{1}}{\left(u_{2,0}-u_{0,0}\right)^{2}}+\frac{h_{-2} h}{\left(u_{0,0}-u_{-2,0}\right)^{2}}\right)+\frac{2}{h_{-1}}\left(K^{(1)} K_{-1}^{(2)}+K^{(2)} K_{-1}^{(1)}\right) .
\end{aligned}
$$

The first and the second densities are the logarithmic residue and the residue of $\tilde{\mathfrak{R}}$, respectively, while $\rho_{2}$ is the residue of the recursion operator $\mathfrak{R}=\tilde{\mathfrak{R}}^{2}(49)$.

According to Proposition 1, the corresponding fluxes are

$$
\begin{aligned}
\sigma_{0}= & \mathcal{S}^{-1}\left(\ln \frac{Q_{u_{1,1}}}{Q_{u_{1,0}}}\right) \\
\sigma_{1}= & \mathcal{S}^{-1}\left(\frac{h_{-1}}{\left(u_{1,0}-u_{-1,0}\right)^{2}} F\right) \\
\sigma_{2}= & \mathcal{S}^{-1}\left(\frac{2 F K^{(1)} K^{(2)}}{h}\right)-\mathcal{S}^{-2}\left(\frac{F h h_{-1}}{\left(u_{2,0}-u_{0,0}\right)^{2}\left(u_{1,0}-u_{-1,0}\right)^{2}}\right) \\
& -\left(1+\mathcal{S}^{-1}\right)\left(\frac{Q_{u_{0,0}}}{Q_{u_{1,0}}} \frac{h_{-1} h_{-2}}{\left(u_{1,0}-u_{-1,0}\right)^{2}\left(u_{0,0}-u_{-2,0}\right)^{2}} \mathcal{S}^{-1} F\right),
\end{aligned}
$$

where

$$
F=\frac{Q_{u_{0,1}} \mathcal{S}^{-1}\left(Q_{u_{1,0}}\right)}{Q_{u_{1,0}} \mathcal{S}^{-1}\left(Q_{u_{1,1}}\right)}-\frac{Q_{u_{0,0}}}{Q_{u_{1,0}}}
$$

and $Q$ is the defining polynomial of equation (1) (or the ABS equations). 
The covariance of the equation allows us to produce three more conservation laws simply by changing $u_{i, 0}$ to $u_{0, i}, h$ to $\hat{h}$ and operator $\mathcal{S}$ to $\mathcal{T}$ in the above densities and fluxes. On the other hand, these conservation laws can also be restricted to the ABS equations in the way described in Remark 1,

For the $\mathrm{H} 1$ equation, its recursion operator (51) is first order and we can use relations (33) to derive some conserved densities. Actually, the following formulas for conserved densities are valid for the equations admitting Hamiltonian operators of zero Laurent order, i.e. equations H1-H3, Q1 and $\mathrm{Q} 3{ }_{\delta=0}$. The conserved densities for all these equations can be written in a relatevly compact form (they are equivalent to the ones given in Proposition 1):

$$
\begin{aligned}
& \varrho_{0}=\ln \left(\mathcal{S}^{-1} f\right)-2 \ln \left(u_{1,0}-u_{-1,0}\right), \\
& \varrho_{1}=\frac{f}{\left(u_{1,0}-u_{-1,0}\right)\left(u_{2,0}-u_{0,0}\right)}, \\
& \varrho_{2}=\left(\varrho_{1}+\mathcal{S}\left(\varrho_{1}\right)\right)^{2}-\mathcal{S}\left(\varrho_{1}\right)^{2}, \\
& \varrho_{3}=\left(\varrho_{1}+\mathcal{S}\left(\varrho_{1}\right)\right)^{3}-\mathcal{S}\left(\varrho_{1}\right)^{3}+3 \varrho_{1} \mathcal{S}\left(\varrho_{1}\right) \mathcal{S}^{2}\left(\varrho_{1}\right), \\
& \varrho_{4}=\left(\varrho_{1}+\mathcal{S}\left(\varrho_{1}\right)\right)^{4}-\mathcal{S}\left(\varrho_{1}\right)^{4}+4 \varrho_{1} \mathcal{S}\left(\varrho_{1}\right) \mathcal{S}^{2}\left(\varrho_{1}\right)\left(\varrho_{1}+2 \mathcal{S}\left(\varrho_{1}\right)+\mathcal{S}^{2}\left(\varrho_{1}\right)+\mathcal{S}^{3}\left(\varrho_{1}\right)\right) .
\end{aligned}
$$

For the H1 equation (50), some of the above densities were found in [23] by the Gardner method.

\section{Conclusions}

The recursion operators presented in this paper produce infinite hierarchies of symmetries and conservation laws for all ABS equations. For the H1 equation we have shown that the proposed recursion operator, $\mathfrak{R}_{H_{1}}$, does satisfy defining equation (16) and thus acting on its symmetry $K^{(1)}$ it will produce its new symmetry $\mathfrak{R}_{H_{1}}\left(K^{(1)}\right)$, provided such action is well defined (i.e. the result belongs to $\mathcal{F}_{Q}$ ). The proof that we can repeatedly apply the recursion operator and each time get a symmetry requires us to investigate the property of Nijenhuis operators [31, 32] and to develop a construction similar to the famous Lenard scheme for the Korteweg-de Vries equation [33, 34. The generalisation of Lenard's scheme for difference equation as well as a complete proof that the operator $\mathfrak{R}$ defined in the Theorem [5 does satisfy (16) for the Viallet equation will be published in our paper [30].

For a quadrilateral equation the first integrability condition has been found by Levi and Yamilov 25. In this paper we have proposed an infinite sequence of integrability conditions in the form of canonical conservation laws. The Levi-Yamilov condition is the first element of this sequence. We have shown that canonical conservation laws are necessary conditions for the existence of a formal recursion operator for a difference equation. As in the case of partial differential equations, cf. [3, 5, 6], one can prove that the existence of a formal recursion operator follows from the existence of an infinite hierarchy of symmetries of increasing order for a difference equation (a general and detailed proof of this assertion will be published elsewhere). There are no doubts that integrability conditions can be successfully used for testing and classification of integrable difference equations (see for example [25]).

Our theory can be extended to difference equations of "higher order", i.e. equations on a rectangular with function $Q$ depending on the set of variables $\left\{u_{p, q} \mid 0 \leq p \leq N, 0 \leq q \leq M\right\}$. For the existence of the elimination map $\mathcal{E}$ we should assume that equation $Q=0$ can be uniquely resolved with respect to the corner variables $u_{0,0}, u_{N, 0}, u_{0, M}$ and $u_{N, M}$. In this case the dynamical 
variables can be defined as

$$
U_{\mathbf{s}}=\left\{u_{n, p} \mid n \in \mathbb{Z}, 0 \leq p<M\right\}, \quad U_{\mathbf{t}}=\left\{u_{q, m} \mid m \in \mathbb{Z}, 0 \leq q<N\right\}, \quad U_{0}=U_{\mathbf{s}} \cup U_{\mathbf{t}} .
$$

We are working on the extension of our theory to the higer order case, the vector case, as well as to the case when $Q$ is not assumed to be an affine-linear polynomial.

\section{Acknowledgments}

We are grateful to V. E. Adler for his clarification about the Viallet equation. Also we would like to thank C. Athorne and A. Pillay for useful discussions. P.X. is supported by the Newton International Fellowship grant NF082473 entitled "Symmetries and integrability of lattice equations and related partial differential equations". A.V.M. and P.X. would like to thank the University of Kent for its hospitality during their visits.

\section{Appendix : The ABS equations}

For every equation in the ABS classification [17] we present its defining polynomial $Q$, the corresponding choices for $a_{i}$ in (11), the corresponding polynomial $f$ and factor $k$ (43), the Hamiltonian operator $\mathcal{H}$ and the seed (root) symmetry of a hierarchy.

The recursion operator for all of the ABS equations can be written as $\mathfrak{R}=\mathcal{H} \circ \mathcal{I}$, where the symplectic operator $\mathcal{I}$ is

$$
\mathcal{I}=\frac{1}{f} \mathcal{S}-\mathcal{S}^{-1} \frac{1}{f}
$$

and the Hamiltonian operator $\mathcal{H}$ is either

$$
\begin{aligned}
\mathcal{H}= & \frac{f_{-1} f f_{1}}{\left(u_{1,0}-u_{-1,0}\right)^{2}\left(u_{2,0}-u_{0,0}\right)^{2}} \mathcal{S}-\mathcal{S}^{-1} \frac{f_{-1} f f_{1}}{\left(u_{1,0}-u_{-1,0}\right)^{2}\left(u_{2,0}-u_{0,0}\right)^{2}} \\
& +2\left(K^{(1)} \mathcal{S}(\mathcal{S}-\mathbf{1})^{-1} K^{(2)}+K^{(2)}(\mathcal{S}-\mathbf{1})^{-1} K^{(1)}\right),
\end{aligned}
$$

which is valid for all of the ABS equations, or the one given in the list for the case of H1-H3, $\mathrm{Q} 1$ and $\mathrm{Q} 3_{\delta=0}$. In (64) and in the lists, $K^{(1)}$ and $K^{(2)}$ denote the first symmetries of the ABS equations, which have the following form

$$
\begin{aligned}
K^{(1)} & =\frac{f}{u_{1,0}-u_{-1,0}}-\frac{1}{2} \partial_{u_{1,0}} f=\frac{f_{-1}}{u_{1,0}-u_{-1,0}}+\frac{1}{2} \partial_{u_{-1,0}} f_{-1}, \\
K^{(2)} & =\frac{f f_{-1}}{\left(u_{1,0}-u_{-1,0}\right)^{2}}\left(\frac{1}{u_{2,0}-u_{0,0}}+\frac{1}{u_{0,0}-u_{-2,0}}\right) .
\end{aligned}
$$

H1

$\begin{array}{ll}\text { Equation } & Q=\left(u_{0,0}-u_{1,1}\right)\left(u_{1,0}-u_{0,1}\right)-\alpha+\beta \\ \text { Parameters } & a_{3}=-a_{5}=1, a_{7}=\beta-\alpha, a_{1}=a_{2}=a_{4}=a_{6}=0 \\ \text { Polynomial } h & f=1 \\ & k=\beta-\alpha \\ \mathcal{H} & \mathcal{H}=K^{(1)}(\mathcal{S}+\mathbf{1})(\mathcal{S}-\mathbf{1})^{-1} K^{(1)} \\ \text { Seed symmetry } & K^{(1)}\end{array}$


$\mathrm{H} 2$

Equation

Parameters

Polynomial $h$

$\mathcal{H}$

Seed symmetry
$Q=\left(u_{0,0}-u_{1,1}\right)\left(u_{1,0}-u_{0,1}\right)+(\beta-\alpha)\left(u_{0,0}+u_{1,0}+u_{0,1}+u_{1,1}\right)-\alpha^{2}+\beta^{2}$

$a_{3}=-a_{5}=1, a_{6}=\beta-\alpha, a_{7}=\beta^{2}-\alpha^{2}, a_{1}=a_{2}=a_{4}=0$

$f=2\left(u_{0,0}+u_{1,0}+\alpha\right)$

$k=\beta-\alpha$

$\mathcal{H}=K^{(1)}(\mathcal{S}+\mathbf{1})(\mathcal{S}-\mathbf{1})^{-1} K^{(1)}-(\mathcal{S}-\mathbf{1})(\mathcal{S}+\mathbf{1})^{-1}$

$K^{(1)}$

\section{H3}

Equation

$Q=\alpha\left(u_{0,0} u_{1,0}+u_{0,1} u_{1,1}\right)-\beta\left(u_{0,0} u_{0,1}+u_{1,0} u_{1,1}\right)+\delta\left(\alpha^{2}-\beta^{2}\right)$

Parameters

$a_{3}=\alpha, a_{5}=-\beta, a_{7}=\delta\left(\alpha^{2}-\beta^{2}\right), a_{1}=a_{2}=a_{4}=a_{6}=0$

Polynomial $h$

$f=u_{0,0} u_{1,0}+\alpha \delta$

$k=\alpha^{2}-\beta^{2}$

$\mathcal{H}$

Seed symmetry

$\mathcal{H}=K^{(1)}(\mathcal{S}+\mathbf{1})(\mathcal{S}-\mathbf{1})^{-1} K^{(1)}-\frac{1}{4} u_{0,0}(\mathcal{S}-\mathbf{1})(\mathcal{S}+\mathbf{1})^{-1} u_{0,0}$ $K^{(1)}$

Q1

Equation

Parameters

$Q=\alpha\left(u_{0,0}-u_{0,1}\right)\left(u_{1,0}-u_{1,1}\right)-\beta\left(u_{0,0}-u_{1,0}\right)\left(u_{0,1}-u_{1,1}\right)+\delta^{2} \alpha \beta(\alpha-\beta)$

Polynomial $h$ $a_{3}=\alpha, a_{4}=\beta-\alpha, a_{5}=-\beta, a_{7}=\delta^{2} \alpha \beta(\alpha-\beta)$

\section{$\mathcal{H}$}

Seed symmetry

$f=\left(\left(u_{0,0}-u_{1,0}\right)^{2}-\alpha^{2} \delta^{2}\right) / \alpha$

$k=\alpha \beta(\beta-\alpha)$

$\mathcal{H}=K^{(1)}(\mathcal{S}+\mathbf{1})(\mathcal{S}-\mathbf{1})^{-1} K^{(1)}-\delta^{2}(\mathcal{S}+\mathbf{1})(\mathcal{S}-\mathbf{1})^{-1}$

\section{Q2}

Equation

$$
\begin{aligned}
& Q=\alpha\left(u_{0,0}-u_{0,1}\right)\left(u_{1,0}-u_{1,1}\right)-\beta\left(u_{0,0}-u_{1,0}\right)\left(u_{0,1}-u_{1,1}\right) \\
& +\alpha \beta(\alpha-\beta)\left(u_{0,0}+u_{1,0}+u_{0,1}+u_{1,1}\right)-\alpha \beta(\alpha-\beta)\left(\alpha^{2}-\alpha \beta+\beta^{2}\right)
\end{aligned}
$$

Parameters

$a_{3}=\alpha, a_{4}=\beta-\alpha, a_{5}=-\beta, a_{6}=\alpha \beta(\alpha-\beta), a_{7}=\alpha \beta(\beta-\alpha)\left(\alpha^{2}-\alpha \beta+\beta^{2}\right)$

Polynomial $h$

$f=\left(\left(u_{0,0}-u_{1,0}\right)^{2}-2 \alpha^{2}\left(u_{0,0}+u_{1,0}\right)+\alpha^{4}\right) / \alpha$

$k=\alpha \beta(\beta-\alpha)$

$\mathcal{H}$ given by (64)

Seed symmetries

$K^{(1)}, K^{(2)}$

\section{Q3}

Equation

$Q=\left(\beta^{2}-\alpha^{2}\right)\left(u_{0,0} u_{1,1}+u_{1,0} u_{0,1}\right)+\beta\left(\alpha^{2}-1\right)\left(u_{0,0} u_{1,0}+u_{0,1} u_{1,1}\right)$

$-\alpha\left(\beta^{2}-1\right)\left(u_{0,0} u_{0,1}+u_{1,0} u_{1,1}\right)-\frac{\delta^{2}\left(\alpha^{2}-\beta^{2}\right)\left(\alpha^{2}-1\right)\left(\beta^{2}-1\right)}{4 \alpha \beta}$

Parameters

$a_{3}=\beta\left(\alpha^{2}-1\right), a_{4}=\beta^{2}-\alpha^{2}, a_{5}=-\alpha\left(\beta^{2}-1\right), a_{7}=-\frac{\delta^{2}\left(\alpha^{2}-\beta^{2}\right)\left(\alpha^{2}-1\right)\left(\beta^{2}-1\right)}{4 \alpha \beta}$

Polynomial $h$

$f=\frac{1}{4 \alpha\left(\alpha^{2}-1\right)}\left(4 \alpha\left(\alpha u_{0,0}-u_{1,0}\right)\left(\alpha u_{1,0}-u_{0,0}\right)-\left(\alpha^{2}-1\right)^{2} \delta^{2}\right)$

$\mathcal{H}$

$k=\left(\alpha^{2}-\beta^{2}\right)\left(\alpha^{2}-1\right)\left(\beta^{2}-1\right)$

Seed symmetries

(i.) $\delta=0: \mathcal{H}=K^{(1)}(\mathcal{S}+\mathbf{1})(\mathcal{S}-\mathbf{1})^{-1} K^{(1)}-\frac{1}{4} u_{0,0}(\mathcal{S}+\mathbf{1})(\mathcal{S}-\mathbf{1})^{-1} u_{0,0}$

(ii.) $\delta \neq 0$ : given by (64)

(i.) $\delta=0: K^{(1)}$; (ii.) $\delta \neq 0: K^{(1)}, K^{(2)}$ 


\section{Q4}

$\begin{array}{ll}\text { Equation } & Q=\operatorname{sn}(\alpha)\left(u_{0,0} u_{1,0}+u_{0,1} u_{1,1}\right)-\operatorname{sn}(\beta)\left(u_{0,0} u_{0,1}+u_{1,0} u_{1,1}\right) \\ & -\operatorname{sn}(\alpha-\beta)\left(u_{0,0} u_{1,1}+u_{1,0} u_{0,1}\right)+k \operatorname{sn}(\alpha) \operatorname{sn}(\beta) \operatorname{sn}(\alpha-\beta)\left(1+u_{0,0} u_{1,0} u_{0,1} u_{1,1}\right) \\ \text { Parameters } & a_{1}=a_{7}=k \operatorname{sn}(\alpha) \operatorname{sn}(\beta) \operatorname{sn}(\alpha-\beta), a_{3}=\operatorname{sn}(\alpha), a_{4}=-\operatorname{sn}(\alpha-\beta), a_{5}=-\operatorname{sn}(\beta) \\ \text { Polynomial } h & f=-k \operatorname{sn}(\alpha)\left(1+u_{0,0}^{2} u_{1,0}^{2}\right)+\frac{1}{\operatorname{sn}(\alpha)}\left(u_{0,0}^{2}+u_{1,0}^{2}-2 \operatorname{cn}(\alpha) \operatorname{dn}(\alpha) u_{0,0} u_{1,0}\right) \\ & k=\operatorname{sn}(\alpha) \operatorname{sn}(\beta) \operatorname{sn}(\beta-\alpha) \\ \mathcal{H} & \text { given by (64) } \\ \text { Seed symmetries } & K^{(1)}, K^{(2)}\end{array}$

\section{References}

[1] Zakharov, V. E. (ed.) : What is integrability? Springer-Verlag, Berlin (1991)

[2] Mikhailov, A. V. (ed.) : Integrability. Lecture Notes in Physics 767, Springer (2009)

[3] Sokolov, V. V., Shabat, A. B.: Classification of integrable evolution equations. In Mathematical Physics Reviews, Vol. 4, of Soviet Sci. Rev. Sect. C: Math. Phys. Rev., pages 221-280. Harwood Academic Publ., Chur (1984)

[4] Mikhailov, A. V., Shabat, A. B., Yamilov, R. I.: Extension of the module of invertible transformations. Classification of integrable systems. Comm. Math. Phys. 115(1),1-19 (1988)

[5] Mikhailov, A. V., Shabat, A. B., Yamilov, R. I.: Symmetry approach to the classification of nonlinear equations. Complete lists of integrable systems. Uspekhi Mat. Nauk 42(4(256)), 3-53 (1987)

[6] Mikhailov, A. V, Shabat, A. B., Sokolov, V. V.: The symmetry approach to classification of integrable equations. In What is integrability?, Springer Ser. Nonlinear Dynamics, pp 115-184. Springer, Berlin (1991)

[7] Sanders, J. A., Wang, J. P.: On the integrability of homogeneous scalar evolution equations. J. Differential Equations 147(2), 410-434 (1998)

[8] Wang, J. P.: Symmetries and Conservation Laws of Evolution Equations. PhD thesis, Vrije Universiteit/Thomas Stieltjes Institute, Amsterdam (1998)

[9] Adler, V. E., Shabat, A. B., Yamilov, R. I.: Symmetry approach to the integrability problem. Theor. Math. Phys. 125, 1603-1661 (2000)

[10] Yamilov, R. I.: Classification of discrete evolution equations. Upsekhi Mat. Nauk 38, 155-156 (1983)

[11] Yamilov, R. I.: Symmetries as integrability criteria for differential difference equations. $J$. Phys. A: Math. Gen. 39, R541-R623 (2006)

[12] Nijhoff, F. W., Capel, H. W.: The discrete Korteweg-de Vries equation. Acta Appl. Math. 39, $133-158(1995)$ 
[13] Grammaticos, B., Halburd, R. G., Ramani, A., Viallet, C-M: How to detect the integrability of discrete systems. J. Phys. A: Math. Theor. 42, 454002 (2009)

[14] Nijhoff, F. W., Walker, A. J.: The discrete and continuous Painlevé hierarchy and the Garnier system. Glasgow Math. J. 43A, 109123 (2001)

[15] Bobenko, A. I., Suris, Yu. B.: Integrable systems on quad-graphs. Int. Math. Res. Notices 11, 573-611 (2002)

[16] Nijhoff, F. W.: Lax pair for the Adler (lattice Krichever-Novikov) system. Phys. Lett. A 297, $49-58(2002)$

[17] Adler, V. E., Bobenko, A. I., Suris, Yu. B.: Classification of integrable equations on quadgraphs. The consistency approach. Comm. Math. Phys. 233, 513-543 (2003)

[18] Adler, V. E., Bobenko, A. I., Suris, Yu. B.: Discrete nonlinear hyperbolic equations. Classification of integrable cases. Funct. Anal. Appl. 43, 3-21 (2009)

[19] Bellon, M. P., Viallet, C. M.: Algebraic entropy. Comm. Math. Phys. 204, 425-437 (1999)

[20] Viallet, C.: Integrable lattice maps: $Q_{V}$, a rational version of $Q_{4}$. Glasgow Math. J. 51A, 157-163 (2009)

[21] Xenitidis, P.: Integrability and symmetries of difference equations: the Adler-Bobenko-Suris case. In Proceedings of the 4th Workshop "Group Analysis of Differential Equations and Integrable Systems" (2009) arXiv: 0902.3954.

[22] Rasin, O. G., Hydon, P. E.: Conservation laws for integrable difference equations. J. Phys. A: Math. Theor. 40, 12763-12773 (2007)

[23] Rasin, A., Schiff, J.: Infinitely many conservation laws for the discrete KdV equation. J. Phys. A: Math. Theor. 42, 175205 (2009)

[24] Rasin, A.: Infinitely many symmetries and conservation laws for quad-graph equations via the Gardner method. arXiv:1001.0724 (2010)

[25] Levi, D., Yamilov, R. I.: The generalized symmetry method for discrete equations. J. Phys. A: Math. Theor. 42, 454012 (2009)

[26] Tongas, A., Tsoubelis, D., Xenitidis, P.: Affine linear and $D_{4}$ symmetric lattice equations: symmetry analysis and reductions. J. Phys. A: Math. Theor. 40, 13353-13384 (2007)

[27] Zhiber, A. V., Sokolov, V. V.: Exactly integrable hyperbolic equations of Liouville type. Uspekhi Mat. Nauk 56(1(337)), 63-106 (2001)

[28] Adler, M.: On a trace functional for formal pseudo differential operators and the symplectic structure of the Korteweg-de Vries type equations. Invent. Math. 50(3), 219-248 (1978/79)

[29] Sanders, J. A., Wang, J. P. : Integrable systems and their recursion operators. Nonlinear Anal. 47, 5213-5240 (2001) 
[30] Mikhailov, A.V., Wang, J. P., Xenitidis, P.: Extended Lenard scheme for difference equations In preparation

[31] Gel'fand, I. M., Dorfman, I. Ya.: Hamiltonian operators and algebraic structures related to them. Funct. Anal. Appl. 13(4), 248-262 (1979)

[32] Fokas, A. S., Fuchssteiner, B.: On the structure of symplectic operators and hereditary symmetries. Lett. Nuovo Cimento (2) 28(8), 299-303 (1980)

[33] Praught, J., Smirnov, G.: Andrew Lenard: A mystery unraveled. SIGMA 1, 005 (2005)

[34] Wang, J. P.: Lenard scheme for two-dimensional periodic Volterra chain. J. Math. Phys. 50, $023506(2009)$ 